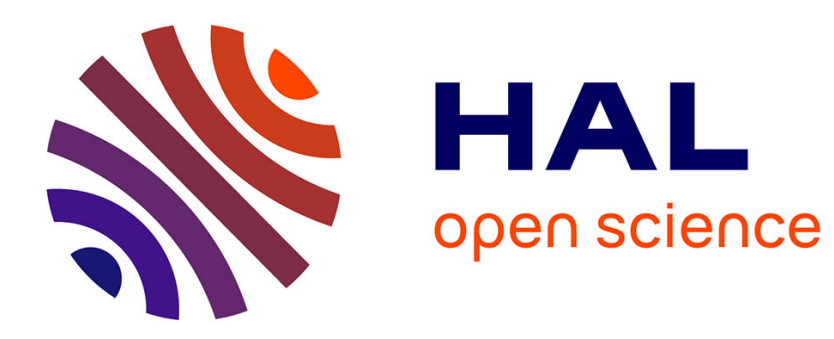

\title{
On the Stability of Self-Propelled Bodies with Respect to their Shape Motion
}

Matthieu Bonnivard

\section{To cite this version:}

Matthieu Bonnivard. On the Stability of Self-Propelled Bodies with Respect to their Shape Motion. 2011. hal-00565408

\author{
HAL Id: hal-00565408 \\ https://hal.science/hal-00565408
}

Preprint submitted on 14 Feb 2011

HAL is a multi-disciplinary open access archive for the deposit and dissemination of scientific research documents, whether they are published or not. The documents may come from teaching and research institutions in France or abroad, or from public or private research centers.
L'archive ouverte pluridisciplinaire HAL, est destinée au dépôt et à la diffusion de documents scientifiques de niveau recherche, publiés ou non, émanant des établissements d'enseignement et de recherche français ou étrangers, des laboratoires publics ou privés. 


\title{
On the stability of self-propelled bodies with respect to their shape motion
}

\author{
Matthieu Bonnivard
}

\author{
Laboratoire de Mathématiques, CNRS UMR 5127, Université de Savoie \\ Campus Scientifique, 73376 Le-Bourget-Du-Lac, France
}

\begin{abstract}
This paper studies the stability of the trajectories of self-propelled bodies immersed in a fluid at low Reynolds number, with respect to their dynamic shape deformation. We consider both adherence and perfect slip boundary conditions on the moving body. Using shape derivative arguments in association with recent higher order regularity results for the Bogovskil operator, we bring the shape stability question to a finite dimension dynamical system analysis.
\end{abstract}

\section{Introduction.}

The locomotion mechanisms of aquatic animals have aroused the interest of zoologists, engineers and mathematicians for a long time. We refer to [24, 27] and the references therein for a review of the works on this subject. Recently, researchers have tried to conceive underwater vehicles that move by shape motion and do not require propellers. A pioneering work in this direction was performed by Tryantafyllou and Tryantafyllou [34]. This type of motion is called self-propelled motion. The principle is the following: the deformation of the object causes a reaction of the fluid, which modifies the inertia and angular momenta of the vehicle and sets the object in motion.

The modelling of self-propelled motion requires to couple the equations of motion of the immersed body with the equations of motion of the fluid. The body is modeled as a deformable solid and its movement is described by Newton's laws of motion. Several works consider a fluid in inviscid and irrotationnal flow, which implies that, after solving an elliptic boundary value problem, one can bring the coupled system to a system of ordinary differential equations involving only the degrees of freedom of the solid. This approach is developed in [7] and [24], for instance. If we take into account the vorticity and viscosity effects, one relevant model for the fluid consists in the stationnary Navier-Stokes equations. This case was studied by San Martín et al. [27], who proved the global existence and uniqueness of the trajectory of a deformable solid on which a smooth deformation is imposed. Nevertheless, at low Reynolds number, the vorticity effects are negligible with respect to the viscosity effects, so the Stokes equations are well adapted to the description of the flow. As a result, Stokes equations are 
relevant to study the locomotion of micro-organisms, such as bacteria or certain species of amœbae. Initiated by the pioneer works of Taylor [33], Berg and Anderson [4] and Purcell [26], the study of swimming at low Reynolds number was the subject of many recent papers. In [29], Shapere and Wilczek introduced a general geometric framework for low Reynolds number swimming, that was developed by Cherman et al. [11] in the two-dimensional case. Let us mention the work of Alouges, DeSimone and Lefebvre [1], who proved the global controllability of a three-sphere swimmer immersed in a Stokes fluid.

In this paper, we consider a simplified model of self-propelled motion at low Reynolds number. This model consists in a deformable solid immersed in a Stokes fluid, in a bounded domain. The displacement of the solid is decomposed into a rigid part corresponding to a global displacement, and a deformation part. We make the assumption that we can impose the deformation and deduce the rigid part from the interaction between the solid and the fluid. The location of the solid-fluid interface is an unknown, so we have to tackle a free boundary problem. This difficulty has been recently investigated in several papers in the case of rigid bodies immersed in a viscous incompressible fluid (see, for instance, Desjardins and Esteban [15], San Martín et al. [28], Feireisl [17] or Gunzburger et al. [19]), and in the case of elastic or viscoelastic bodies (see, for instance, Coutand and Shkoller $[12,13]$, Chambolle et al. [10], Desjardins et al. [16], and Boulakia [6]).

In this work, we give a new proof of the global existence and uniqueness of the trajectory of a deformable solid on which the nonrigid part of the displacement is imposed, which is immersed in a viscous incompressible fluid at low Reynolds number. The proof given in [27] for a general viscous incompressible flow is based on the transformation of a non-cylindrical problem into a cylindrical one, and on a fixed point argument. The principle of our proof is similar to the approach adopted by Munnier [24] in the case of a potential flow: we use the existence, uniqueness and regularity of the solution to the Stokes problem to bring the coupled system of equations to a system of ordinary differential equations. To prove the regularity of the corresponding operator, we need to establish the differentiability of the weak solution to the Stokes problem with respect to both rigid and nonrigid deformations of the solid, and to the boundary conditions at the solid-fluid interface. To this aim, we use shape derivative methods that were introduced by Murat and Simon [25] and Sokołowski and Zolésio [31]. Several difficulties arise here, due to the fact that we treat both classical adherence conditions and perfect slip conditions which are not frequent in the literature. Besides, to deal with boundary conditions that depend on the deformation of the solid, we need high order regularity results for the Bogovskii operator which were recently proved by Mitrea et al. [23].

One major interest of our approach is that, treating the deformation as a parameter is the differential system, we are able to prove the stability of the trajectories of the solid with respect to the imposed deformation. The stability of the trajectories is a crucial step to perform a dynamical shape control of the immersed body. Although this goal is not achieved, it was the first motivation of this work. A way to deal with this problem is to impose a perfect slip condition at the solid-fluid interface, to consider small boundary deformations and to produce dynamic boundary conditions by the rugosity effect (see Casado-Díaz et al. [9], Bucur et al. [8]).

The paper is organized as follows. After presenting the model in Section 2, we state our 
main results in Section 3. The proof to Theorem 1 is carried out in Section 4, and Theorem 2 is proved in Section 5.

\section{Presentation of the model.}

All results in this paper are valid in two or three dimensions. For the sake of simplicity we present a two-dimensional model, which is adapted from [27]. However, the derivation of the model and the mathematical study we perform in the next sections can be adapted to the three-dimensional case without extra fundamental mathematical difficulties.

\subsection{A kinematic model of self-propelled motion in a fluid.}

We denote by $\Omega$ the domain occupied by the solid-fluid system, which is supposed to be a bounded and regular open set. The state of the system at the initial instant is called the reference configuration. We denote by $\mathcal{S}_{0} \subset \Omega\left(\right.$ resp. $\mathcal{F}_{0}:=\Omega \backslash \overline{\mathcal{S}_{0}}$ ) the domain occupied by the solid (resp. the fluid) in the reference configuration. We suppose that $\partial \mathcal{S}_{0}$ is of class $\mathcal{C}^{3}$. We assume that the solid is at positive distance from the boundary of $\Omega$ in the reference configuration. Thus, there exists $\delta>0$ such that if we define the following subset of $\Omega$ :

$$
\Omega_{-\delta}=\{x \in \Omega \mid \operatorname{dist}(x, \partial \Omega)>\delta\}
$$

then

$$
\mathcal{S}_{0} \subset \Omega_{-\delta} .
$$

We consider a reference frame with the origin at the mass center of $\mathcal{S}_{0}$, and a mobile frame attached to the solid and which coincides with the reference frame at instant $t=0$. In what follows, we use the following convention. We reserve the letters $x, y$ for space variables in the reference frame, while $x^{*}$ stands for a space variable in the mobile frame. We write with a star the vector fields whose components are expressed in the mobile frame.

We impose a smooth deformation of the solid, that is, we consider a time-dependant vector field $\Theta^{*} \in \mathcal{C}^{1}\left([0, \infty), \mathcal{C}^{3}\left(\overline{\mathcal{S}_{0}}, \mathbb{R}^{2}\right)\right)$ such that the deformation field $X^{*}$ in the solid is defined by

$$
X^{*}(y, t)=y+\Theta^{*}(y, t), \quad(y, t) \in \overline{\mathcal{S}_{0}} \times[0, \infty) .
$$

We suppose that the deformation satisfies the following hypothesis:

(H1) For every $t \geqslant 0$, the mapping $y \rightarrow X^{*}(y, t)$ is a $\mathcal{C}^{3}$ diffeomorphism from $\mathcal{S}_{0}$ onto $\mathcal{S}^{*}(t):=X^{*}\left(\mathcal{S}_{0}, t\right)$.

For every $t \geqslant 0$ we denote by $Y^{*}(\cdot, t)$ the inverse of $X^{*}(\cdot, t)$. We define the deformation velocity of the solid in Eulerian coordinates by

$$
w^{*}\left(x^{*}, t\right)=\frac{\partial \Theta^{*}}{\partial t}\left(Y^{*}\left(x^{*}, t\right), t\right) \quad t \geqslant 0, \quad x^{*} \in \mathcal{S}^{*}(t) .
$$

We suppose that the volume of the solid is preserved during its movement, which leads to the following constraint on $w^{*}$ : 
(H2) $\int_{\partial \mathcal{S}^{*}(t)} w^{*}\left(x^{*}, t\right) \mathrm{d} s\left(x^{*}\right)=0, \quad t \geqslant 0$.

Here we have denoted $\mathrm{d} s$ the surface measure on $\partial \mathcal{S}^{*}(t)$.

Since we aim to study self-propelled motions, it is natural to assume that the deformation $X^{*}$ does not affect the linear and angular momenta of the solid. The expression of these properties requires to define the density field $\rho^{*}$ of the solid. Let $\rho_{0}$ be the density field of the solid in the reference configuration $\mathcal{S}_{0}$. The local form of the mass conservation in the solid yields the following expression of $\rho^{*}$ :

$$
\rho^{*}\left(x^{*}, t\right)=\frac{\rho_{0}\left(Y^{*}\left(x^{*}, t\right)\right)}{\operatorname{det}\left(\nabla X^{*}\right)\left(Y^{*}\left(x^{*}, t\right), t\right)} \quad t \geqslant 0, \quad x^{*} \in \mathcal{S}^{*}(t) .
$$

Since the origin of the reference system of coordinates coincides with the mass center of $\mathcal{S}_{0}$, the conservation of the linear and angular momenta yields the following constraints on $w^{*}$ :

(H3) $\int_{\mathcal{S}^{*}(t)} \rho^{*}\left(x^{*}, t\right) w^{*}\left(x^{*}, t\right) \mathrm{d} x^{*}=0, \quad t \geqslant 0$,

(H4) $\int_{\mathcal{S}^{*}(t)} \rho^{*}\left(x^{*}, t\right) x^{* \perp} \cdot w^{*}\left(x^{*}, t\right) \mathrm{d} x^{*}=0, \quad t \geqslant 0$,

where for every $x=\left(x_{1}, x_{2}\right) \in \mathbb{R}^{2}$ we denote by $x^{\perp}$ the vector $x^{\perp}=\left(-x_{2}, x_{1}\right)$.

Let $X$ denote the motion of the solid. We assume that there exists a rigid displacement $X^{\mathrm{R}}$ such that

$$
X(y, t)=X^{\mathrm{R}}\left(X^{*}(y, t), t\right), \quad t \geqslant 0, \quad y \in \mathcal{S}_{0} .
$$

The rigid motion $X^{\mathrm{R}}: \mathbb{R}^{2} \times[0, \infty) \rightarrow \mathbb{R}^{2}$ is given by

$$
X^{\mathrm{R}}\left(x^{*}, t\right)=\xi(t)+R_{\theta(t)} x^{*}
$$

where, for every $t \geqslant 0, \xi(t)$ is the position of the mass center of the solid, $\theta(t)$ is the angle giving the orientation of the solid with respect to a fixed axis in the reference frame and $R_{\theta(t)} \in S O_{2}(\mathbb{R})$ is the matrix associated to the rotation of angle $\theta(t)$. We denote by $\mathcal{S}(\xi(t), \theta(t), t)$ the domain occupied by the solid at instant $t$, that is,

$$
\mathcal{S}(\xi(t), \theta(t), t)=R_{\theta(t)} \mathcal{S}^{*}(t)+\xi(t), \quad t \geqslant 0 .
$$

The Eulerian velocity field of the solid is given by

$$
u^{S}(x, t)=\xi^{\prime}(t)+\theta^{\prime}(t)(x-\xi(t))^{\perp}+w(x, t), \quad t \geqslant 0, \quad x \in \mathcal{S}(\xi(t), \theta(t), t),
$$

where

$$
w(x, t)=R_{\theta(t)} w^{*}\left(R_{-\theta(t)}(x-\xi(t)), t\right), \quad t \geqslant 0, \quad x \in \mathcal{S}(\xi(t), \theta(t), t) .
$$

The Eulerian density field of the body is given by

$$
\rho(x, t)=\rho^{*}\left(R_{-\theta(t)}(x-\xi(t)), t\right), \quad t \geqslant 0, \quad x \in \mathcal{S}(\xi(t), \theta(t), t),
$$

with $\rho^{*}$ given by (2). The mass $m$ of the body and its moment of inertia $I(t)$ with respect to an axis orthogonal to the plane of motion and passing by the mass center of $\mathcal{S}(\xi(t), \theta(t), t)$, are given by

$$
m=\int_{\mathcal{S}(\xi(t), \theta(t), t)} \rho(x, t) \mathrm{d} x
$$




$$
I(t)=\int_{\mathcal{S}(\xi(t), \theta(t), t)} \rho(x, t)|x-\xi(t)|^{2} \mathrm{~d} x, \quad t \geqslant 0 .
$$

Let us notice that from (2), (3), (4) and (5), we have

$$
\begin{gathered}
m=\int_{\mathcal{S}_{0}} \rho_{0}(y) \mathrm{d} y, \\
I(t)=\int_{\mathcal{S}_{0}} \rho_{0}(y)\left|X^{*}(y, t)\right|^{2} \mathrm{~d} y, \quad t \geqslant 0 .
\end{gathered}
$$

In particular, since $X^{*} \in \mathcal{C}^{1}\left(\overline{\mathcal{S}_{0}} \times[0, \infty), \Omega\right)$, the mapping $t \geqslant 0 \rightarrow I(t) \in \mathbb{R}$ is of class $\mathcal{C}^{1}$.

\subsection{The full system.}

If there is no risk of confusion, we denote

$$
\mathcal{S}(t)=\mathcal{S}(\xi(t), \theta(t), t)
$$

the domain occupied by the solid at instant $t$, and we define

$$
\mathcal{F}(t)=\Omega \backslash \overline{\mathcal{S}(t)}
$$

the domain filled by the fluid at instant $t$. We denote $\mathcal{F}_{0}=\mathcal{F}(0)$. For $k=1,2$ we define

$$
\mathbb{H}^{k}(\mathcal{F}(t))=H^{k}(\mathcal{F}(t))^{2}
$$

where $H^{k}(\mathcal{F}(t))$ is the usual Sobolev space of order $k$. We denote by $L_{0}^{2}(\mathcal{F}(t))$ the subspace of $L^{2}(\mathcal{F}(t))$ consisting of functions with mean value equal to 0 .

We consider the following problem: find a real $T>0$ and functions $\xi \in \mathcal{C}^{2}\left((0, T), \mathbb{R}^{2}\right) \cap$ $\mathcal{C}^{1}\left([0, T), \mathbb{R}^{2}\right)$ and $\theta \in \mathcal{C}^{2}((0, T), \mathbb{R}) \cap \mathcal{C}^{1}([0, T), \mathbb{R})$ solutions to the system

$$
\begin{array}{r}
m \xi^{\prime \prime}(t)=-\int_{\partial \mathcal{S}(t)} \sigma(u, p) n \mathrm{~d} s, \quad t \in(0, T), \\
\left(\mathrm{I} \theta^{\prime}\right)^{\prime}(t)=-\int_{\partial \mathcal{S}(t)} \sigma(u, p) n \cdot(x-\xi)^{\perp} \mathrm{d} s, \quad t \in(0, T),
\end{array}
$$

where for every $t \in(0, T),(u, p) \in \mathbb{H}^{1}(\mathcal{F}(t)) \times L_{0}^{2}(\mathcal{F}(t))$ is solution to the following problem:

$$
\begin{aligned}
-\nu \Delta u+\nabla p=0 & \text { in } \mathcal{F}(t), \\
\operatorname{div} u=0 & \text { in } \mathcal{F}(t), \\
u=0 & \text { on } \partial \Omega, \\
u \cdot n=u^{S} \cdot n & \text { on } \partial \mathcal{S}(t), \\
{[\sigma(u, p) n]_{\text {tan }}=0 } & \text { on } \partial \mathcal{S}(t) .
\end{aligned}
$$

In the above system $\nu>0$ stands for the viscosity of the fluid, $u$ and $p$ denote the Eulerian velocity field and the pressure field of the fluid, respectively. $n$ stands for the unit normal 
to $\partial \mathcal{S}(t)$ oriented towards the solid. In (6)-(7), a prime stands for the derivation operator with respect to time. The stress tensor field $\sigma$ is defined by

$$
\sigma(u, p)=2 \nu D(u)-p \mathrm{Id}
$$

where $D(u)$ is the symmetric part of $\nabla u$ defined by

$$
D(u)=\frac{1}{2}\left((\nabla u)+(\nabla u)^{T}\right)
$$

and Id is the identity matrix in $M_{2}(\mathbb{R})$.

Let us point out that the integrals in (6)-(7) require the $H^{1}$ regularity of $\sigma$ in the neighborhood of $\partial \mathcal{S}(t)$, so that $\sigma n$ is properly defined in $L^{2}(\partial \mathcal{S}(t))$, in the sense of trace. This regularity will be discussed in Section 4 .

Notice that (11)-(12) express a perfect slip condition at the solid-fluid interface. All the results in this paper hold if we replace (11)-(12) by the adherence condition

$$
u=u^{S} \quad \text { on } \partial \mathcal{S}(t)
$$

System (6)-(12) is completed by the initial conditions

$$
\begin{array}{r}
\xi(0)=0, \theta(0)=0, \\
\xi^{\prime}(0)=\dot{\xi}_{0}, \theta^{\prime}(0)=\dot{\theta}_{0} .
\end{array}
$$

\section{Statement of the main results.}

We follow the notations in Section 2. Let us first define the set $\mathcal{D}$ of admissible deformations $\Theta^{*}$.

Let $K \in(0,1)$ be a given constant. Since $\mathcal{C}^{3}\left(\overline{\mathcal{S}_{0}}, \mathbb{R}^{2}\right)$ is a Banach space, (H1) holds for every $\Theta^{*} \in \mathcal{C}^{1}\left([0, \infty), \mathcal{C}^{3}\left(\overline{\mathcal{S}_{0}}, \mathbb{R}^{2}\right)\right)$ satisfying the following condition:

$$
\forall t \geqslant 0 \quad\left\|\Theta^{*}(\cdot, t)\right\|_{\left.\mathcal{C}^{3}\left(\overline{\mathcal{S}_{0}}, \mathbb{R}^{2}\right)\right)} \leqslant K
$$

Replacing $K$ by a smaller positive value if necessary, we may assume that for every $\Theta^{*} \in$ $\mathcal{C}^{1}\left([0, \infty), \mathcal{C}^{3}\left(\overline{\mathcal{S}_{0}}, \mathbb{R}^{2}\right)\right)$ satisfying $(15)$, the following property holds:

$$
\forall t \geqslant 0 \quad \mathcal{S}^{*}(t) \subset \Omega_{-\delta / 2}
$$

The set $\mathcal{D}$ of admissible deformations is defined by

$$
\begin{aligned}
\mathcal{D}:= & \left\{\Theta^{*} \in \mathcal{C}^{1}\left([0, \infty), \mathcal{C}^{3}\left(\overline{\mathcal{S}_{0}}, \mathbb{R}^{2}\right)\right) \quad \mid \quad \forall t \geqslant 0\right. \\
& \left\|\Theta^{*}(t)\right\|_{\left.\mathcal{C}^{3}\left(\overline{\mathcal{S}_{0}}, \mathbb{R}^{2}\right)\right)} \leqslant K, \quad \int_{\partial \mathcal{S}^{*}(t)} w^{*}\left(x^{*}, t\right) \mathrm{d} s\left(x^{*}\right)=0, \\
& \left.\int_{\mathcal{S}^{*}(t)} \rho^{*}\left(x^{*}, t\right) w^{*}\left(x^{*}, t\right) \mathrm{d} x^{*}=0, \quad \int_{\mathcal{S}^{*}(t)} \rho^{*}\left(x^{*}, t\right) x^{* \perp} \cdot w^{*}\left(x^{*}, t\right) \mathrm{d} x^{*}=0 .\right\}
\end{aligned}
$$

Theorem 1. Let $\left(\dot{\xi}_{0}, \dot{\theta}_{0}\right) \in \mathbb{R}^{3}$ and $\Theta^{*} \in \mathcal{D}$. There exists $T_{\max }>0$ such that: 
- For every $T \in\left(0, T_{\max }\right)$, system (6)-(12), completed with initial conditions (14), admits one unique solution on $[0, T]$.

- The following alternative holds:

(i) $T_{\max }=\infty$; or

(ii) $\lim _{t \rightarrow T_{\text {max }}} \operatorname{dist}(\mathcal{S}(t), \partial \Omega)=0$.

- In the two-dimensional case, if the adherence condition (13) is imposed at the solidfluid interface, then $T_{\max }=\infty$.

In the second result, $\mathcal{D}$ is endowed with the $\mathcal{C}^{1}\left([0, \infty), \mathcal{C}^{3}\left(\overline{\mathcal{S}_{0}}, \mathbb{R}^{2}\right)\right)$ norm.

Theorem 2. Let $\left(\dot{\xi}_{0}, \dot{\theta}_{0}\right) \in \mathbb{R}^{3}$ and $\Theta^{*} \in \mathcal{D}$. Let $(\xi, \theta)$ be the maximal solution to (6)-(12) associated to $\Theta^{*}$, with initial conditions (14). Let $\left(\Theta_{n}^{*}\right)_{n \in \mathbb{N}}$ be a sequence of deformations converging to $\Theta^{*}$ in $\mathcal{D}$. For every $n \in \mathbb{N}$ we denote by $\left(\xi_{n}, \theta_{n}\right)$ the maximal solution associated to $\Theta_{n}^{*}$ with the same initial conditions. Then:

- There exists $T_{1}>0$ such that $(\xi, \theta),\left(\xi_{n}, \theta_{n}\right)$ exist on $\left[0, T_{1}\right]$ for all $n \in \mathbb{N}$.

- The sequence $\left(\xi_{n}, \theta_{n}\right)_{n \in \mathbb{N}}$ converges to $(\xi, \theta)$ uniformly in $\mathcal{C}^{1}\left(\left[0, T^{*}\right], \mathbb{R}^{3}\right)$, for any time $T^{*}$ such that the solution $\left(\xi_{n}, \theta_{n}\right)$ is well-defined on $\left[0, T^{*}\right]$ for any $n$.

\section{Proof of Theorem 1.}

In this section, the deformation $\Theta^{*} \in \mathcal{D}$ is given. To obtain the local existence and uniqueness of the trajectories, we rewrite problem (6)-(12) as a system of second order differential equations satisfied by the unknown functions $\xi, \theta$, and apply the Cauchy-Lipschitz theorem.

Step 1 . For every $t \geqslant 0$ and every $(\xi, \theta) \in \mathbb{R}^{3}$ we denote by $\mathcal{S}(\xi, \theta, t)$ the domain

$$
\mathcal{S}(\xi, \theta, t)=\xi+R_{\theta} \mathcal{S}^{*}(t) .
$$

By (16), there exists an open subset $\mathcal{W} \subset \mathbb{R}^{3}$ such that $0 \in \mathcal{W}$ and

$$
\forall t \geqslant 0, \forall(\xi, \theta) \in \mathcal{W}, \quad \overline{\mathcal{S}(\xi, \theta, t)} \subset \Omega .
$$

For every $t \geqslant 0$ and every $(\xi, \theta) \in \mathcal{W}$ we denote by $\mathcal{F}(\xi, \theta, t)$ the domain

$$
\mathcal{F}(\xi, \theta, t)=\Omega \backslash \overline{\mathcal{S}(\xi, \theta, t)} .
$$

We denote by $\alpha$ a quadruplet $(\xi, \theta, \dot{\xi}, \dot{\theta})$ in $\mathbb{R}^{6}$ and by $\mathcal{U}$ the admissible set for the parameter $\alpha$, defined by

$$
\mathcal{U}=\mathcal{W} \times \mathbb{R}^{3} .
$$

For every $(\alpha, t) \in \mathcal{U} \times[0, \infty)$ we define $\left(u_{\alpha, t}, p_{\alpha, t}\right)$ in $\mathbb{H}^{1}(\mathcal{F}(\xi, \theta, t)) \times L_{0}^{2}(\mathcal{F}(\xi, \theta, t))$ as the solution to the following problem:

$$
\left\{\begin{aligned}
-\nu \Delta u_{\alpha, t}+\nabla p_{\alpha, t}=0 & \text { in } \mathcal{F}(\xi, \theta, t) \\
\operatorname{div} u_{\alpha, t}=0 & \text { in } \mathcal{F}(\xi, \theta, t) \\
u_{\alpha, t}=0 & \text { on } \partial \Omega \\
u_{\alpha, t} \cdot n_{\xi, \theta, t}=u_{\alpha, t}^{S} \cdot n_{\xi, \theta, t} & \text { on } \partial \mathcal{S}(\xi, \theta, t) \\
{\left[\sigma\left(u_{\alpha, t}, p_{\alpha, t}\right) n_{\xi, \theta, t}\right]_{\tan }=0 } & \text { on } \partial \mathcal{S}(\xi, \theta, t) \\
7 &
\end{aligned}\right.
$$


where $n_{\xi, \theta, t}$ stands for the unit normal to $\partial \mathcal{S}(\xi, \theta, t)$ oriented towards $\mathcal{S}(\xi, \theta, t)$. The function $u_{\alpha, t}^{S}$ is defined by

$$
u_{\alpha, t}^{S}(x)=\dot{\xi}+\dot{\theta}(x-\xi)^{\perp}+R_{\theta} w^{*}\left(R_{-\theta}(x-\xi), t\right), \quad x \in \partial \mathcal{S}(\xi, \theta, t),
$$

and $L_{0}^{2}(\mathcal{F}(\xi, \theta, t))$ is the subspace of $L^{2}(\mathcal{F}(\xi, \theta, t))$ consisting of functions with mean value equal to 0 .

Let us precise the sense of (19). For every $(\xi, \theta, t) \in \mathcal{W} \times[0, \infty)$, we define the following subspace of $\mathbb{H}^{1}(\mathcal{F}(\xi, \theta, t))$ :

$$
\mathbb{H}_{\text {tan }}^{1}(\mathcal{F}(\xi, \theta, t))=\left\{\psi \in \mathbb{H}^{1}(\mathcal{F}(\xi, \theta, t)): \psi \cdot n_{\xi, \theta, t}=0 \text { on } \partial \mathcal{F}(\xi, \theta, t), \quad \psi=0 \text { on } \partial \Omega\right\} .
$$

$\left(u_{\alpha, t}, p_{\alpha, t}\right) \in \mathbb{H}^{1}(\mathcal{F}(\xi, \theta, t)) \times L_{0}^{2}(\mathcal{F}(\xi, \theta, t))$ is solution to (19) in the following sense:

$$
\operatorname{div} u_{\alpha, t}=0 \quad \text { in } L^{2}(\mathcal{F}(\xi, \theta, t)),
$$

$u_{\alpha, t}$ satisfies the boundary conditions

$$
u_{\alpha, t}=0 \quad \text { on } \partial \Omega, \quad u_{\alpha, t} \cdot n_{\xi, \theta, t}=u_{\alpha, t}^{S} \cdot n_{\xi, \theta, t} \quad \text { on } \partial \mathcal{S}(\xi, \theta, t)
$$

in the sense of trace, and for every test function $\psi \in \mathbb{H}_{\text {tan }}^{1}(\mathcal{F}(\xi, \theta, t))$,

$$
\nu \int_{\mathcal{F}(\xi, \theta, t)} \nabla u_{\alpha, t}: \nabla \psi \mathrm{d} x-\int_{\mathcal{F}(\xi, \theta, t)} p_{\alpha, t}(\operatorname{div} \psi) \mathrm{d} x=0 .
$$

To state the local $\mathbb{H}^{2} \times H^{1}$ regularity of $\left(u_{\alpha, t}, p_{\alpha, t}\right)$ in the neighborhood of $\partial \mathcal{S}(\xi, \theta, t)$, we introduce an open subset $\Omega^{\prime} \subset \mathbb{R}^{2}$ such that

$$
\mathcal{S}_{0} \subset \Omega^{\prime} \subset \Omega
$$

and $\partial \Omega^{\prime}$ is at positive distance from $\partial \mathcal{S}_{0}$ and $\partial \Omega$. We set

$$
\mathcal{F}_{0}^{\prime}=\mathcal{F}_{0} \cap \Omega^{\prime}
$$

By classic regularity results for the Stokes problem with slip boundary conditions (see for instance [32], [2]), problem (19) admits one unique solution $\left(u_{\alpha, t}, p_{\alpha, t}\right)$ in $\mathbb{H}^{1}(\mathcal{F}(\xi, \theta, t)) \times$ $L_{0}^{2}(\mathcal{F}(\xi, \theta, t))$. Furthermore, this solution satisfies

$$
\left(u_{\alpha, t}, p_{\alpha, t}\right) \in \mathbb{H}^{2}\left(X_{\xi, \theta, t}\left(\mathcal{F}_{0}^{\prime}\right)\right) \times H^{1}\left(X_{\xi, \theta, t}\left(\mathcal{F}_{0}^{\prime}\right)\right)
$$

We now define the operators

$$
F: \mathcal{U} \times[0, \infty) \rightarrow \mathbb{R}^{2}, \quad G: \mathcal{U} \times[0, \infty) \rightarrow \mathbb{R}
$$

by

$$
\left\{\begin{array}{l}
F(\alpha, t)=-\frac{1}{m} \int_{\partial \mathcal{S}(\xi, \theta, t)} \sigma\left(u_{\alpha, t}, p_{\alpha, t}\right) n_{\xi, \theta, t} \mathrm{~d} s \\
G(\alpha, t)=-\frac{1}{\mathrm{I}(t)}\left(\int_{\partial \mathcal{S}(\xi, \theta, t)} \sigma\left(u_{\alpha, t}, p_{\alpha, t}\right) n_{\xi, \theta, t} \cdot(x-\xi)^{\perp} \mathrm{d} s+\mathrm{I}^{\prime}(t) \dot{\theta}\right) .
\end{array}\right.
$$


With these notations, problem (6)-(12), completed by initial conditions (14), can be rewritten as follows: find $T>0$ and functions $\xi \in \mathcal{C}^{2}\left((0, T), \mathbb{R}^{2}\right) \cap \mathcal{C}^{1}\left([0, T), \mathbb{R}^{2}\right)$ and $\theta \in$ $\mathcal{C}^{2}((0, T), \mathbb{R}) \cap \mathcal{C}^{1}([0, T), \mathbb{R})$ solutions to the system

$$
\begin{aligned}
\left(\xi^{\prime \prime}(t), \theta^{\prime \prime}(t)\right) & =(F, G)\left(\xi(t), \theta(t), \xi^{\prime}(t), \theta^{\prime}(t), t\right), \quad t \in(0, T), \\
\left(\xi(0), \theta(0), \xi^{\prime}(0), \theta^{\prime}(0)\right) & =\left(0,0, \dot{\xi}_{0}, \dot{\theta}_{0}\right) .
\end{aligned}
$$

Remark 3. Since the dependance of $\mathcal{S}(\xi, \theta, t), n_{\xi, \theta, t}$ and $\left(u_{\alpha, t}, p_{\alpha, t}\right)$ on the time parameter $t$ appears only through $\Theta^{*}(t)$ and $\left(\Theta^{*}\right)^{\prime}(t)\left(\right.$ via $\mathcal{S}^{*}(t)$ and the boundary conditions on $\partial \mathcal{S}(\xi, \theta, t)$ in (19)), system (25) could be written as

$$
\begin{aligned}
\left(\xi^{\prime \prime}(t), \theta^{\prime \prime}(t)\right) & =(F, G)\left(\xi(t), \theta(t), \xi^{\prime}(t), \theta^{\prime}(t), \Theta^{*}(t),\left(\Theta^{*}\right)^{\prime}(t)\right), \quad t \in(0, T) \\
\left(\xi(0), \theta(0), \xi^{\prime}(0), \theta^{\prime}(0)\right) & =\left(0,0, \dot{\xi}_{0}, \dot{\theta}_{0}\right) .
\end{aligned}
$$

In this formulation, the couple $\left(\Theta^{*}(t),\left(\Theta^{*}\right)^{\prime}(t)\right)$ appears as the natural control variable for system (26). However, since we aim to study the stability of the solid-fluid system with respect to deformations $\Theta^{*} \in \mathcal{D}$, which is an infinite-dimensional function space, the analyticity of $(F, G)$ with respect to $\left(\Theta^{*}(t),\left(\Theta^{*}\right)^{\prime}(t)\right)$ is not well defined, to our knowledge. For this reason, and to simplify the notations, we keep formulation (25) in the rest of the paper.

Step 2. To obtain the continuity of F, G and the local Lipschitz condition with respect to $\alpha$, we need to prove the regularity of the solution to (19) with respect to $(\alpha, t)$. To this end, we introduce a $\mathcal{C}^{3}$-diffeomorphism $X_{\xi, \theta, t}$ from $\Omega$ onto itself, which is defined in the next paragraph.

The principle is to extend the deformation $X^{*}$ up to a neighborhood of $\mathcal{S}_{0}$ and to combine this deformation with the flow of a Cauchy problem, so that the restriction of $X_{\xi, \theta, t}$ to $\mathcal{S}_{0}$ coincides with the effective deformation of the solid, namely,

$$
X_{\xi, \theta, t}(y)=\xi+R_{\theta} X^{*}(y, t), \quad y \in \mathcal{S}_{0} .
$$

For every $t \geqslant 0$, we define $\Theta^{*}(t) \in \mathcal{C}^{3}\left(\overline{\mathcal{S}_{0}}, \mathbb{R}^{2}\right)$ by

$$
\Theta^{*}(t)=\Theta^{*}(\cdot, t)
$$

We consider an operator of extension

$$
\mathcal{E}: \mathcal{C}^{3}\left(\overline{\mathcal{S}_{0}}, \mathbb{R}^{2}\right) \rightarrow \mathcal{C}^{3}\left(\Omega, \mathbb{R}^{2}\right)
$$

which is supposed to be linear and bounded, and satisfies

$$
\left(\mathcal{E} \Theta^{*}(t)\right)(y)=\Theta^{*}(y, t), \quad \Theta^{*} \in \mathcal{D}, \quad y \in \overline{\mathcal{S}_{0}} .
$$

The existence of $\mathcal{E}$ results from the regularity of $\partial \mathcal{S}_{0}$. Let $\tau$ be a $\mathcal{C}^{\infty}$ function with compact support in $\Omega_{-\delta / 2}$, that is equal to 1 in a neighborhood of $\Omega_{-\delta}$. We define the extended deformation $\overline{X^{*}}$ by

$$
\overline{X^{*}}(y, t)=y+\left(\mathcal{E} \Theta^{*}(t)\right)(y) \tau(y) \quad t \geqslant 0, \quad y \in \Omega .
$$


By definition of $\mathcal{E}$ and $\tau$, and since $\Theta^{*} \in \mathcal{D}$, the mapping $\overline{X^{*}} \in \mathcal{C}^{1}\left([0, \infty), \mathcal{C}^{3}\left(\Omega, \mathbb{R}^{2}\right)\right)$. Moreover, by continuity of $\mathcal{E}$, supposing that the constant $K$ that appears in the definition of $\mathcal{D}$ is small enough, for every $t \geqslant 0$, the mapping $\overline{X^{*}}(\cdot, t)$ is a $\mathcal{C}^{3}$-diffeomorphism from $\mathcal{F}_{0}$ onto $\mathcal{F}^{*}(t):=\Omega \backslash \overline{\mathcal{S}^{*}(t)}$ such that

$$
\forall y \in \mathcal{F}_{0} \quad \operatorname{dist}(y, \partial \Omega)<\delta / 2 \Rightarrow \overline{X^{*}}(y, t)=y .
$$

To deal with the rigid part of the displacement, for every $(\xi, \theta) \in \mathcal{W}$, we introduce $\chi_{\xi, \theta}$ the flow of the following Cauchy problem:

$$
\left\{\begin{aligned}
\frac{\mathrm{d} \chi_{\xi, \theta}}{\mathrm{d} s}(z, s) & =z_{\xi, \theta}\left(\chi_{\xi, \theta}(z, s), s\right), \\
\chi_{\xi, \theta}(z, 0) & =z, \quad z \in \Omega
\end{aligned}\right.
$$

where the function $z_{\xi, \theta}$ is defined for every $(x, s) \in \mathbb{R}^{2} \times \mathbb{R}_{+}$by

$$
z_{\xi, \theta}(x, s)=\left(\xi+\theta(x-s \xi)^{\perp}\right) \tau(x) .
$$

Since $z_{\xi, \theta}$ is $\mathcal{C}^{\infty}$ and for every $s \geqslant 0, z_{\xi, \theta}(\cdot, s)$ has a compact support in $\Omega$, problem (30) admits a unique solution on the interval $[0,1]$. One can easily check that for every $z \in \mathcal{S}_{0}$, this solution is equal to

$$
\chi_{\xi, \theta}(z, s)=s \xi+R_{s \theta} z, \quad s \in[0,1] .
$$

Since the mapping

$$
(x, s, \xi, \theta) \in \mathbb{R}^{2} \times \mathbb{R}_{+} \times \mathcal{W} \rightarrow z_{\xi, \theta}(x, s) \in \mathbb{R}^{2}
$$

is of class $\mathcal{C}^{\infty}$, the mapping

$$
(z, \xi, \theta) \in \Omega \times \mathcal{W} \rightarrow \chi_{\xi, \theta}(z, 1) \in \Omega
$$

is of class $\mathcal{C}^{\infty}$. Since $z_{\xi, \theta}$ has a compact support in $\Omega, \chi_{\xi, \theta}(\cdot, 1)$ is a $\mathcal{C}^{\infty}$-diffeomorphism from $\Omega$ onto itself. For every $(\xi, \theta, t) \in \mathcal{W} \times[0, \infty)$ we now define

$$
X_{\xi, \theta, t}(y)=\chi_{\xi, \theta}\left(\overline{X^{*}}(y, t), 1\right) \quad y \in \Omega .
$$

Applying (31) with $s=1$, we get that $X_{\xi, \theta, t}$ satisfies $(27)$. Thus, for every $(\xi, \theta, t) \in$ $\mathcal{W} \times[0, \infty)$, the restriction of $X_{\xi, \theta, t}$ to $\mathcal{F}_{0}$ is a $\mathcal{C}^{3}$-diffeomorphism from $\mathcal{F}_{0}$ onto $\mathcal{F}(\xi, \theta, t)$. By composition, the mapping

$$
(\xi, \theta, t, y) \in \mathcal{W} \times \mathcal{F}_{0} \rightarrow X_{\xi, \theta, t}(y) \in \mathbb{R}^{2}
$$

is of class $\mathcal{C}^{\infty}\left(\mathcal{W}, \mathcal{C}^{1}\left([0, \infty), \mathcal{C}^{3}\left(\overline{\mathcal{F}_{0}}, \mathbb{R}^{2}\right)\right)\right)$.

The crucial point in the proof of Theorem 1 is the following:

Proposition 4. The mapping

$$
(\alpha, t) \in \mathcal{U} \times[0, \infty) \rightarrow \sigma\left(u_{\alpha, t}, p_{\alpha, t}\right) \circ X_{\xi, \theta, t} \in H^{1}\left(\mathcal{F}_{0}^{\prime}\right)^{4}
$$

is of class $\mathcal{C}^{1}$. 
Proof of Proposition 4. The proof is based on technics developed by Simon [30] and Bello et al. [3] to prove the differentiability of the solution to a Stokes (resp. Navier-Stokes) problem, with respect to Lipschitz deformations of the domain. The principle is to transform a problem with constant coefficients posed on a moving domain, into a problem with variable coefficients posed on a fixed reference domain, via an appropriate change of unknown functions. Then the implicit function theorem is applied. The main difference with all previous works is that we impose no-slip boundary conditions, which requires to consider the variation of the normal on $\partial \mathcal{S}_{0}$ induced by the diffeomorphism $X_{\xi, \theta, t}$. Besides, the boundary conditions are inhomogeneous and depend on the parameters $\alpha$ and $t$. To deal with these boundary conditions we use a lift operator to transform the nonhomogeneous problem into an homogeneous problem with a source term. Another difficulty arises here: in order to preserve the $\mathbb{H}^{2} \times H^{1}$ regularity of the solution in a neighborhood of the solid, we need a lift operator involving Sobolev spaces of higher order that the well-known Bogovskii operator (see for instance [5] or [18]). The existence of such operators, defined on a subspace of $\mathbb{H}^{3 / 2}$ with value in $\mathbb{H}^{1}$, was proved recently by Mitrea, Mitrea and Monniaux [23].

For every $(\xi, \theta, t) \in \mathcal{W} \times[0, \infty)$ we denote by $J_{\xi, \theta, t} \in \mathcal{C}^{2}\left(\overline{\mathcal{F}_{0}}, \mathbb{R}\right)^{4}$ the Jacobian matrix of the restriction of $X_{\xi, \theta, t}$ to $\mathcal{F}_{0}$. Using properties (29) and (33) and the Taylor formulas, we obtain the following

Lemma 5. The mapping

$$
(\xi, \theta, t) \in \mathcal{W} \times[0, \infty) \rightarrow J_{\xi, \theta, t} \in W^{2, \infty}\left(\mathcal{F}_{0}\right)^{4}
$$

is of class $\mathcal{C}^{1}$.

In the next paragraph, we use the restriction of $X_{\xi, \theta, t}$ to $\mathcal{F}_{0}$ to write problem (19) in the reference domain $\mathcal{F}_{0}$. We denote by $\mathcal{J}_{\xi, \theta, t}$ the determinant of $J_{\xi, \theta, t}$. We can assume without loss of generality that

$$
\mathcal{J}_{\xi, \theta, t}(y)>0 \quad \forall(\xi, \theta, t, y) \in \mathcal{W} \times[0, \infty) \times \mathcal{F}_{0}
$$

Recall that $\mathcal{F}_{0}^{\prime}$ is defined by $(22)$. The change of functions that we use is motivated by the following result:

Lemma 6. Let $(\xi, \theta, t) \in \mathcal{W} \times[0, \infty)$ and $(u, p) \in \mathbb{H}^{1}(\mathcal{F}(\xi, \theta, t)) \times L^{2}(\mathcal{F}(\xi, \theta, t))$. Let $(U, P) \in$ $\mathbb{H}^{1}\left(\mathcal{F}_{0}\right) \times L^{2}\left(\mathcal{F}_{0}\right)$ defined by

$$
U=\mathcal{J}_{\xi, \theta, t} J_{\xi, \theta, t}^{-1}\left(u \circ X_{\xi, \theta, t}\right), \quad P=\mathcal{J}_{\xi, \theta, t}\left(p \circ X_{\xi, \theta, t}\right) .
$$

The following results hold:

(i) $p \in L_{0}^{2}(\mathcal{F}(\xi, \theta, t))$ if and only if $P \in L_{0}^{2}\left(\mathcal{F}_{0}\right)$,

(ii) $(u, p) \in \mathbb{H}^{2}\left(X_{\xi, \theta, t}\left(\mathcal{F}_{0}^{\prime}\right)\right) \times H^{1}\left(X_{\xi, \theta, t}\left(\mathcal{F}_{0}^{\prime}\right)\right)$ if and only if $(U, P) \in \mathbb{H}^{2}\left(\mathcal{F}_{0}^{\prime}\right) \times H^{1}\left(\mathcal{F}_{0}^{\prime}\right)$,

(iii) $\operatorname{div} u=0$ in $L^{2}\left(X_{\xi, \theta, t}\left(\mathcal{F}_{0}\right)\right)$ if and only if $\operatorname{div} U=0$ in $L^{2}\left(\mathcal{F}_{0}\right)$. 
Proof of Lemma 6. To prove (i), notice that by change of variable $x=X_{\xi, \theta, t}(y)$,

$$
\begin{aligned}
\int_{\mathcal{F}(\xi, \theta, t)} p(x) \mathrm{d} x & =\int_{\mathcal{F}_{0}} \mathcal{J}_{\xi, \theta, t}(y)\left(p \circ X_{\xi, \theta, t}\right)(y) \mathrm{d} y \\
& =\int_{\mathcal{F}_{0}} P(y) \mathrm{d} y .
\end{aligned}
$$

Point (ii) results from the fact that the coefficients of $J_{\xi, \theta, t}$ and $J_{\xi, \theta, t}^{-1}$ are in $W^{2, \infty}\left(\mathcal{F}_{0}\right)$ and that $X_{\xi, \theta, t}$ is in $\mathcal{C}^{2}\left(\overline{\mathcal{F}_{0}}, \mathbb{R}^{2}\right)$. To prove (iii), we perform the following calculation. For every $\zeta \in \mathcal{C}_{c}^{1}(\mathcal{F}(\xi, \theta, t))$, using Green's formula, change of variable $x=X_{\xi, \theta, t}(y)$ and the chain rule formula

$$
\nabla\left(\zeta \circ X_{\xi, \theta, t}\right)(y)=\left[J_{\xi, \theta, t}(y)\right]^{T} \nabla \zeta\left(X_{\xi, \theta, t}(y)\right), \quad y \in \mathcal{F}_{0},
$$

we obtain that

$$
\begin{aligned}
\int_{\mathcal{F}(\xi, \theta, t)}(\operatorname{div} u)(x) \zeta(x) \mathrm{d} x & =-\int_{\mathcal{F}(\xi, \theta, t)} u(x) \cdot \nabla \zeta(x) \mathrm{d} x \\
& =-\int_{\mathcal{F}_{0}}\left(u \circ X_{\xi, \theta, t}\right)(y) \cdot\left(\left[J_{\xi, \theta, t}(y)^{-1}\right]^{T} \nabla\left(\zeta \circ X_{\xi, \theta, t}\right)(y)\right) \mathcal{J}_{\xi, \theta, t}(y) \mathrm{d} y \\
& =-\int_{\mathcal{F}_{0}} \mathcal{J}_{\xi, \theta, t}(y)\left(\left[J_{\xi, \theta, t}(y)^{-1}\right]\left(u \circ X_{\xi, \theta, t}\right)(y)\right) \cdot \nabla\left(\zeta \circ X_{\xi, \theta, t}\right)(y) \mathrm{d} y \\
& =\int_{\mathcal{F}_{0}}(\operatorname{div} U)(y)\left(\zeta \circ X_{\xi, \theta, t}\right)(y) \mathrm{d} y .
\end{aligned}
$$

Since the mapping

$$
\zeta \in \mathcal{C}_{c}^{1}(\mathcal{F}(\xi, \theta, t)) \rightarrow \zeta \circ X_{\xi, \theta, t} \in \mathcal{C}_{c}^{1}\left(\mathcal{F}_{0}\right)
$$

is bijective, this proves (iii) by density in $L^{2}$ of the $\mathcal{C}^{1}$ functions with compact support.

For every $\alpha=(\xi, \theta, \dot{\xi}, \dot{\theta}) \in \mathcal{U}$ and every $t \geqslant 0$, we define the following change of functions:

$$
U_{\alpha, t}=\mathcal{J}_{\xi, \theta, t} J_{\xi, \theta, t}^{-1}\left(u_{\alpha, t} \circ X_{\xi, \theta, t}\right), \quad P_{\alpha, t}=\mathcal{J}_{\xi, \theta, t}\left(p_{\alpha, t} \circ X_{\xi, \theta, t}\right),
$$

where $\left(u_{\alpha, t}, p_{\alpha, t}\right) \in \mathbb{H}^{1}(\mathcal{F}(\xi, \theta, t)) \times L_{0}^{2}(\mathcal{F}(\xi, \theta, t))$ is the solution to (19). This change of functions was introduced by Inoue and Wakimoto [22] in the case $\mathcal{J}_{\xi, \theta, t} \equiv 1$. By (23) and Lemma $6,\left(U_{\alpha, t}, P_{\alpha, t}\right)$ has the following regularity:

$$
\left(U_{\alpha, t}, P_{\alpha, t}\right) \in \mathbb{H}^{2}\left(\mathcal{F}_{0}^{\prime}\right) \cap \mathbb{H}^{1}\left(\mathcal{F}_{0}\right) \times H^{1}\left(\mathcal{F}_{0}^{\prime}\right) \cap L_{0}^{2}\left(\mathcal{F}_{0}\right) .
$$

We will prove that $\left(u_{\alpha, t}, p_{\alpha, t}\right)$ is solution to (19) if and only $\left(U_{\alpha, t}, P_{\alpha, t}\right)$ is solution to the following problem:

$$
\left\{\begin{aligned}
\sum_{i}\left(-\nu \operatorname{div}\left(L_{i}(\xi, \theta, t) U_{\alpha, t}\right)+\sum_{j} \partial_{j}\left(M_{i j}(\xi, \theta, t) P_{\alpha, t}\right)\right) \nabla X_{\xi, \theta, t}^{(i)} & =0 & & \text { in } \mathcal{F}_{0}, \\
\operatorname{div} U_{\alpha, t} & =0 & & \text { in } \mathcal{F}_{0}, \\
U_{\alpha, t} & =0 & & \text { on } \partial \Omega, \\
U_{\alpha, t} \cdot n_{0} & =U_{\alpha, t}^{S} \cdot n_{0} & & \text { on } \partial \mathcal{S}_{0}, \\
{\left[\sum_{i}\left[\left(L_{i}(\xi, \theta, t) U_{\alpha, t}\right) \cdot n_{0}\right] \nabla X_{\xi, \theta, t}^{(i)}\right]_{t a n} } & =0 & &
\end{aligned}\right.
$$


in a sense that will be made precise below. In the above system, $n_{0}$ stands for the unit normal to $\partial \mathcal{S}_{0}$ oriented towards $\mathcal{S}_{0}$. For every $(\alpha, t) \in \mathcal{U} \times[0, \infty), U_{\alpha, t}^{S} \in \mathcal{C}^{3}\left(\overline{\mathcal{S}_{0}}, \mathbb{R}^{2}\right)$ is defined by

$$
U_{\alpha, t}^{S}(y)=\mathcal{J}_{\xi, \theta, t}(y)\left(D X_{t}^{*}(y)\right)^{-1}\left(R_{\theta}^{-1} \dot{\xi}+\dot{\theta} X^{*}(y, t)^{\perp}+\frac{\partial X^{*}}{\partial t}(y, t)\right), \quad y \in \mathcal{S}_{0}
$$

where $D X_{t}^{*}$ stands for the Jacobian matrix of $X^{*}(\cdot, t)$. Notice that

$$
J_{\xi, \theta, t}(y)=R_{\theta} D X_{t}^{*}(y), \quad y \in \mathcal{S}_{0} .
$$

$M(\xi, \theta, t) \in W^{2, \infty}\left(\mathcal{F}_{0}\right)^{4}$ is defined by

$$
M(\xi, \theta, t)(y)=\left[J_{\xi, \theta, t}(y)^{-1}\right]^{T}, \quad y \in \mathcal{F}_{0} .
$$

For $i=1,2, L_{i}(\xi, \theta, t) \in \mathcal{L}\left(\mathbb{H}^{2}\left(\mathcal{F}_{0}^{\prime}\right) \cap \mathbb{H}^{1}\left(\mathcal{F}_{0}\right), \mathbb{H}^{1}\left(\mathcal{F}_{0}^{\prime}\right) \cap L^{2}\left(\mathcal{F}_{0}\right)^{2}\right)$ is the bounded operator defined by

$$
L_{i}(\xi, \theta, t) U=\mathcal{J}_{\xi, \theta, t} J_{\xi, \theta, t}^{-1} M(\xi, \theta, t) \nabla\left(\mathcal{J}_{\xi, \theta, t}^{-1} \nabla X_{\xi, \theta, t}^{(i)} \cdot U\right) \quad \forall U \in \mathbb{H}^{2}\left(\mathcal{F}_{0}^{\prime}\right) \cap \mathbb{H}^{1}\left(\mathcal{F}_{0}\right) .
$$

Let us precise the sense of (37). We define the following subspace of $\mathbb{H}^{1}\left(\mathcal{F}_{0}\right)$ :

$$
\mathbb{H}_{\text {tan }}^{1}\left(\mathcal{F}_{0}\right)=\left\{\phi \in \mathbb{H}^{1}\left(\mathcal{F}_{0}\right): \phi \cdot n_{0}=0 \text { on } \partial \mathcal{F}_{0}, \quad \phi=0 \text { on } \partial \Omega\right\} .
$$

$\left(U_{\alpha, t}, P_{\alpha, t}\right) \in \mathbb{H}^{1}\left(\mathcal{F}_{0}\right) \times L_{0}^{2}\left(\mathcal{F}_{0}\right)$ satisfies $(37)$ in the following sense:

$$
\operatorname{div} U_{\alpha, t}=0 \quad \text { in } L^{2}\left(\mathcal{F}_{0}\right),
$$

$U_{\alpha, t}$ satisfies the boundary conditions

$$
U_{\alpha, t}=0 \quad \text { on } \partial \Omega, \quad U_{\alpha, t} \cdot n_{0}=U_{\alpha, t}^{S} \cdot n_{0} \quad \text { on } \partial \mathcal{S}_{0}
$$

in the sense of trace, and for every test function $\phi \in \mathbb{H}_{\text {tan }}^{1}\left(\mathcal{F}_{0}\right)$,

$$
\begin{aligned}
& \nu \int_{\mathcal{F}_{0}} \sum_{i}\left[\left(L_{i}(\xi, \theta, t) U_{\alpha, t}\right) \cdot \nabla\left(\nabla X_{\xi, \theta, t}^{(i)} \cdot \phi\right)\right](y) \mathrm{d} y \\
- & \int_{\mathcal{F}_{0}} P_{\alpha, t}(y) \sum_{i}\left[\left(M(\xi, \theta, t) \nabla\left(\nabla X_{\xi, \theta, t}^{(i)} \cdot \phi\right)\right)(y)\right]^{(i)} \mathrm{d} y=0 .
\end{aligned}
$$

To prove that problems (19) and (37) are equivalent via transformation (35), we need the following result involving the spaces of test functions $\mathbb{H}_{\text {tan }}^{1}\left(\mathcal{F}_{0}\right)$ and $\mathbb{H}_{\text {tan }}^{1}(\mathcal{F}(\xi, \theta, t)$ ) (defined by (20), p. 8).

Lemma 7. For every $\psi \in \mathbb{H}_{\text {tan }}^{1}(\mathcal{F}(\xi, \theta, t))$, if we define the function $\phi: \mathcal{F}_{0} \rightarrow \mathbb{R}^{2}$ by

$$
\phi(y)=J_{\xi, \theta, t}(y)^{-1}\left(\psi \circ X_{\xi, \theta, t}\right)(y), \quad y \in \mathcal{F}_{0},
$$

then $\phi \in \mathbb{H}_{\text {tan }}^{1}\left(\mathcal{F}_{0}\right)$, and the mapping

$$
\psi \in \mathbb{H}_{\text {tan }}^{1}(\mathcal{F}(\xi, \theta, t)) \rightarrow \phi \in \mathbb{H}_{\text {tan }}^{1}\left(\mathcal{F}_{0}\right)
$$

is one-to-one and onto. 
Proof of Lemma 7. Let $\psi \in \mathbb{H}^{1}(\mathcal{F}(\xi, \theta, t))$. Clearly, by the regularity of $X_{\xi, \theta, t}$, the function $\phi$ defined by $(41)$ belongs to $\mathbb{H}^{1}\left(\mathcal{F}_{0}\right)$ and vanishes on $\partial \Omega$ in the sense of trace. We need to prove the following equivalence:

$$
\psi \cdot n_{\xi, \theta, t}=0 \quad \text { on } \partial \mathcal{S}(\xi, \theta, t) \quad \Longleftrightarrow \quad \phi \cdot n_{0}=0 \quad \text { on } \partial \mathcal{S}_{0}
$$

By density of $C^{1}(\overline{\mathcal{F}(\xi, \theta, t)})$ in $\mathbb{H}^{1}(\mathcal{F}(\xi, \theta, t))$, we may assume that $\psi \in C^{1}(\overline{\mathcal{F}(\xi, \theta, t)})$. Introducing a parametrisation $\gamma$ of $\partial \mathcal{S}_{0}$ and the corresponding parametrisation $X_{\xi, \theta, t} \circ \gamma$ of $\partial \mathcal{S}_{\xi, \theta, t}$, one can express the unit normal $n_{\xi, \theta, t}$ as a function of $n_{0}$ and of the first order space derivatives of $X_{\xi, \theta, t}$ as follows:

$$
n_{\xi, \theta, t}\left(X_{\xi, \theta, t}(y)\right)=\frac{\left[J_{\xi, \theta, t}(y)^{-1}\right]^{T} n_{0}(y)}{\left|\left[J_{\xi, \theta, t}(y)^{-1}\right]^{T} n_{0}(y)\right|}, \quad y \in \mathcal{F}_{0} .
$$

Consequently, for every $y \in \partial \mathcal{S}_{0}$,

$$
\begin{aligned}
\left(\psi \circ X_{\xi, \theta, t}\right)(y) \cdot n_{\xi, \theta, t}\left(X_{\xi, \theta, t}(y)\right)=0 & \Longleftrightarrow\left(\psi \circ X_{\xi, \theta, t}\right)(y) \cdot\left(\left[J_{\xi, \theta, t}(y)^{-1}\right]^{T} n_{0}(y)\right)=0 \\
& \Longleftrightarrow\left(J_{\xi, \theta, t}(y)^{-1}\left(\psi \circ X_{\xi, \theta, t}\right)(y)\right) \cdot n_{0}(y)=0 \\
& \Longleftrightarrow \phi(y) \cdot n_{0}(y)=0 .
\end{aligned}
$$

This proves (42).

Let us prove that for every $\phi \in \mathbb{H}_{\text {tan }}^{1}\left(\mathcal{F}_{0}\right)$, if we define $\psi \in \mathbb{H}_{\text {tan }}^{1}\left(\mathcal{F}_{0}\right)$ by $(41)$, then $(40)$ is equivalent to (21). Noticing that for $i=1,2$,

$$
\psi^{(i)} \circ X_{\xi, \theta, t}=\nabla X_{\xi, \theta, t}^{(i)} \cdot \phi
$$

and applying formule (34) to $\zeta=\psi^{(i)} \circ X_{\xi, \theta, t}$, we obtain the following chain rule:

$$
\nabla \psi^{(i)}\left(X_{\xi, \theta, t}(y)\right)=\left(M(\xi, \theta, t) \nabla\left(\nabla X_{\xi, \theta, t}^{(i)} \cdot \phi\right)\right)(y), \quad y \in \mathcal{F}_{0}
$$

By change of variables $x=X_{\xi, \theta, t}(y)$, we obtain

$$
\begin{aligned}
& \int_{\mathcal{F}(\xi, \theta, t)} \sum_{i} \nabla u_{\alpha, t}^{(i)}(x) \cdot \nabla \psi^{(i)}(x) \mathrm{d} x \\
= & \int_{\mathcal{F}_{0}} \sum_{i}\left(\mathcal{J}_{\xi, \theta, t} M(\xi, \theta, t) \nabla\left(\nabla X_{\xi, \theta, t}^{(i)} \cdot\left(\mathcal{J}_{\xi, \theta, t}^{-1} U_{\alpha, t}\right)\right)\right)(y) \cdot\left(M(\xi, \theta, t) \nabla\left(\nabla X_{\xi, \theta, t}^{(i)} \cdot \phi\right)\right)(y) \mathrm{d} y \\
= & \int_{\mathcal{F}_{0}} \sum_{i}\left[\left(L_{i}(\xi, \theta, t) U_{\alpha, t}\right) \cdot \nabla\left(\nabla X_{\xi, \theta, t}^{(i)} \cdot \phi\right)\right](y) \mathrm{d} y
\end{aligned}
$$

and

$$
\begin{aligned}
& \int_{\mathcal{F}(\xi, \theta, t)} p_{\alpha, t}(x)(\operatorname{div} \psi)(x) \mathrm{d} x \\
= & \int_{\mathcal{F}_{0}} P_{\alpha, t}(y) \sum_{i}\left[\left(M(\xi, \theta, t) \nabla\left(\nabla X_{\xi, \theta, t}^{(i)} \cdot \phi\right)\right)(y)\right]^{(i)} \mathrm{d} y .
\end{aligned}
$$

By Lemma 7, we conclude that problems (19) and (37) are equivalent. 
Remark 8. System (37) can be obtained from equation (40) by the following formal computations. If $\left(U_{\alpha, t}, P_{\alpha, t}\right)$ is regular enough, then for every $\phi \in \mathbb{H}_{\text {tan }}^{1}\left(\mathcal{F}_{0}\right)$,

$$
\begin{aligned}
& \int_{\mathcal{F}_{0}} \sum_{i}\left[\left(L_{i}(\xi, \theta, t) U_{\alpha, t}\right) \cdot \nabla\left(\nabla X_{\xi, \theta, t}^{(i)} \cdot \phi\right)\right](y) \mathrm{d} y \\
= & -\int_{\mathcal{F}_{0}} \sum_{i}\left[\left(\operatorname{div}\left(L_{i}(\xi, \theta, t) U_{\alpha, t}\right)\right)(y) \nabla X_{\xi, \theta, t}^{(i)}(y)\right] \cdot \phi(y) \mathrm{d} y \\
+ & \int_{\partial \mathcal{S}_{0}} \sum_{i}\left[\left(\left(L_{i}(\xi, \theta, t) U_{\alpha, t}\right)(y) \cdot n_{0}(y)\right) \nabla X_{\xi, \theta, t}^{(i)}(y)\right] \cdot \phi(y) \mathrm{d} s(y)
\end{aligned}
$$

and

$$
\begin{aligned}
& -\int_{\mathcal{F}_{0}} P_{\alpha, t}(y) \sum_{i}\left[\left(M(\xi, \theta, t) \nabla\left(\nabla X_{\xi, \theta, t}^{(i)} \cdot \phi\right)\right)(y)\right]^{(i)} \mathrm{d} y \\
= & \int_{\mathcal{F}_{0}} \sum_{i j}\left[\partial_{j}\left(M_{i j}(\xi, \theta, t) P_{\alpha, t}\right)(y) \nabla X_{\xi, \theta, t}^{(i)}(y)\right] \cdot \phi(y) \mathrm{d} y \\
& -\int_{\partial \mathcal{S}_{0}} P(y) \sum_{i j}\left[M_{i j}(y) n_{0}^{(j)}(y) \nabla X_{\xi, \theta, t}^{(i)}(y)\right] \cdot \phi(y) \mathrm{d} s(y) .
\end{aligned}
$$

Using definition (38), we obtain

$$
\begin{aligned}
& \int_{\partial \mathcal{S}_{0}} P(y) \sum_{i j}\left(M_{i j}(y) n_{0}^{(j)}(y) \nabla X_{\xi, \theta, t}^{(i)}(y)\right) \cdot \phi(y) \mathrm{d} s(y) \\
= & \int_{\partial \mathcal{S}_{0}} P(y)\left(M(\xi, \theta, t)(y) n_{0}(y)\right) \cdot\left(J_{\xi, \theta, t}(y) \phi(y)\right) \mathrm{d} s(y) \\
= & \int_{\partial \mathcal{S}_{0}} P(y) n_{0}(y) \cdot \phi(y) \mathrm{d} s(y) \\
= & 0 .
\end{aligned}
$$

To prove the regularity of the solution to problem (37) with respect to $(\alpha, t)$, we need to lift the boundary condition. To this end, we introduce the following subspace of $H^{3 / 2}\left(\partial \mathcal{S}_{0}\right)^{2}$ :

$$
\mathcal{G}\left(\partial \mathcal{S}_{0}\right)=\left\{\Gamma \in H^{3 / 2}\left(\partial \mathcal{S}_{0}\right)^{2} \mid \int_{\partial \mathcal{S}_{0}} \Gamma \cdot n_{0} \mathrm{~d} s=0\right\}
$$

We consider a bounded operator

$$
\Pi: \mathcal{G}\left(\partial \mathcal{S}_{0}\right) \rightarrow \mathbb{H}^{2}\left(\mathcal{F}_{0}\right)
$$

such that for every $\Gamma \in \mathcal{G}\left(\partial \mathcal{S}_{0}\right)$, if we define

$$
K=\Pi \Gamma,
$$


then $K$ satisfies the following constraints:

$$
\left\{\begin{array}{rll}
\operatorname{div} K=0 & \text { in } \mathcal{F}_{0} \\
K=0 & \text { on } \partial \Omega \\
K=\Gamma & \text { on } \partial \mathcal{S}_{0}
\end{array}\right.
$$

The existence of $\Pi$ is proved in [23] (Corollary 1.4). For every $(\alpha, t) \in \mathcal{U} \times[0, \infty)$, we define $K_{\alpha, t} \in \mathbb{H}^{2}\left(\mathcal{F}_{0}\right)$ by

$$
K_{\alpha, t}=\Pi U_{\alpha, t} .
$$

Since $\Pi$ is bounded and since the mapping

$$
(\alpha, t) \in \mathcal{U} \times[0, \infty) \rightarrow U_{\alpha, t}^{S} \in H^{3 / 2}\left(\partial \mathcal{S}_{0}\right)^{2}
$$

is of class $\mathcal{C}^{1}$, by composition, we get the following

Lemma 9. The mapping

$$
(\alpha, t) \in \mathcal{U} \times[0, \infty) \rightarrow K_{\alpha, t} \in \mathbb{H}^{2}\left(\mathcal{F}_{0}\right)
$$

is of class $\mathcal{C}^{1}$.

We define the new unknown function

$$
V_{\alpha, t}:=U_{\alpha, t}-K_{\alpha, t}
$$

$V_{\alpha, t} \in \mathbb{H}^{2}\left(\mathcal{F}_{0}^{\prime}\right) \cap \mathbb{H}_{\text {tan }}^{1}\left(\mathcal{F}_{0}\right)$ and $\left(U_{\alpha, t}, P_{\alpha, t}\right)$ is solution to $(37)$ if and only if $\left(V_{\alpha, t}, P_{\alpha, t}\right)$ is solution to

$$
\left\{\begin{aligned}
\sum_{i}\left[-\nu \operatorname{div}\left(L_{i}(\xi, \theta, t)\left[V_{\alpha, t}+K_{\alpha, t}\right]\right)+\sum_{j} \partial_{j}\left(M_{i j}(\xi, \theta, t) P_{\alpha, t}\right)\right] \nabla X_{\xi, \theta, t}^{(i)}= & 0 \text { in } \mathcal{F}_{0} \\
\operatorname{div} V_{\alpha, t}= & 0 \text { in } \mathcal{F}_{0}
\end{aligned}\right.
$$

Using (46) we can write $\left(V_{\alpha, t}, P_{\alpha, t}\right)$ as a zero of a bounded operator depending on the parameter $(\alpha, t) \in \mathcal{U} \times[0, \infty)$. The definition of this operator requires to introduce the following Banach spaces:

$$
\begin{aligned}
& A=\mathbb{H}^{2}\left(\mathcal{F}_{0}^{\prime}\right) \cap \mathbb{H}_{\text {tan }}^{1}\left(\mathcal{F}_{0}\right) \times H^{1}\left(\mathcal{F}_{0}^{\prime}\right) \cap L_{0}^{2}\left(\mathcal{F}_{0}\right), \\
& B=L^{2}\left(\mathcal{F}_{0}^{\prime}\right)^{2} \cap\left(\mathbb{H}_{\text {tan }}^{1}\right)^{\prime}\left(\mathcal{F}_{0}\right) \times H^{1}\left(\mathcal{F}_{0}^{\prime}\right) \cap L_{0}^{2}\left(\mathcal{F}_{0}\right),
\end{aligned}
$$

endowed with the norms:

$$
\begin{aligned}
\|(V, P)\|_{A} & =\|V\|_{\mathbb{H}^{2}\left(\mathcal{F}_{0}^{\prime}\right)}+\|V\|_{\mathbb{H}^{1}\left(\mathcal{F}_{0}\right)}+\|P\|_{H^{1}\left(\mathcal{F}_{0}^{\prime}\right)}+\|P\|_{L^{2}\left(\mathcal{F}_{0}\right)} \\
\|(f, g)\|_{B} & =\|f\|_{L^{2}\left(\mathcal{F}_{0}^{\prime}\right)^{2}}+\|f\|_{\left(\mathbb{H}_{\text {tan }}^{1}\right)^{\prime}\left(\mathcal{F}_{0}\right)}+\|g\|_{H^{1}\left(\mathcal{F}_{0}^{\prime}\right)}+\|g\|_{L^{2}\left(\mathcal{F}_{0}\right)}
\end{aligned}
$$

In the definition of $B,\left(\mathbb{H}_{\text {tan }}^{1}\right)^{\prime}\left(\mathcal{F}_{0}\right)$ stands for the dual space of $\mathbb{H}_{\text {tan }}^{1}\left(\mathcal{F}_{0}\right)$. We now define the operator

$$
H: \mathcal{U} \times[0, \infty) \times A \rightarrow B
$$

by

$$
\left\{\begin{array}{l}
H(\alpha, t ; V, P)=(Q(\alpha, t ; V, P), R(\alpha, t ; V, P)), \\
Q(\alpha, t ; V, P)=\sum_{i}\left[-\nu \operatorname{div}\left(L_{i}(\xi, \theta, t)\left[V+K_{\alpha, t}\right]\right)+\sum_{j} \partial_{j}\left(M_{i j}(\xi, \theta, t) P\right)\right] \nabla X_{\xi, \theta, t}^{(i)}, \\
R(\alpha, t ; V, P)=\operatorname{div} V .
\end{array}\right.
$$


Remark 10. For every $V \in \mathbb{H}_{\text {tan }}^{1}\left(\mathcal{F}_{0}\right)$, div $V \in L_{0}^{2}\left(\mathcal{F}_{0}\right)$, which justifies the definition of $B$. Indeed, by Stokes formula,

$$
\begin{aligned}
\int_{\mathcal{F}_{0}} \operatorname{div} V \mathrm{~d} x & =\int_{\partial \mathcal{S}_{0}} V \cdot n_{0} \mathrm{~d} s \\
& =0
\end{aligned}
$$

With these notations, system (46) can be rewritten in the following implicit form:

$$
H\left(\alpha, t ; V_{\alpha, t}, P_{\alpha, t}\right)=0 .
$$

The final step is to prove that the mapping

$$
(\alpha, t) \in \mathcal{U} \times[0, \infty) \rightarrow\left(V_{\alpha, t}, P_{\alpha, t}\right)
$$

solution to (48) is of class $\mathcal{C}^{1}$. For this purpose, we apply the implicit function theorem. Let $\left(\alpha^{*}, t^{*}\right)$ be a given point in $\mathcal{U} \times[0, \infty)$, with $\alpha^{*}=\left(\xi^{*}, \dot{\xi}^{*}, \theta^{*}, \dot{\theta}^{*}\right)$. We introduce the following notations:

$$
\begin{aligned}
& X=X_{\xi^{*}, \theta^{*}, t^{*}}, J=J_{\xi^{*}, \theta^{*}, t^{*}}, \mathcal{J}=\mathcal{J}_{\xi^{*}, \theta^{*}, t^{*}}, \\
& M^{*}=M\left(\xi^{*}, \theta^{*}, t^{*}\right), L_{i}^{*}=L_{i}\left(\xi^{*}, \theta^{*}, t^{*}\right), \\
& \mathcal{F}=X\left(\mathcal{F}_{0}\right), \mathcal{F}^{\prime}=X\left(\mathcal{F}_{0}^{\prime}\right), \\
& \left(V^{*}, P^{*}\right)=\left(V_{\alpha^{*}, t^{*}}, P_{\alpha^{*}, t^{*}}\right) .
\end{aligned}
$$

We will apply the implicit function theorem to the function $H$ defined by (47), at point $\left(\alpha^{*}, t^{*}, V^{*}, P^{*}\right) \in \mathcal{U} \times[0, \infty) \times A$.

- $H$ is a $\mathcal{C}^{1}$ mapping from $\mathcal{U} \times[0, \infty) \times A$ into $B$. Indeed,

- since the mappings $(\xi, \theta, t) \in \mathcal{W} \times[0, \infty) \rightarrow J_{\xi, \theta, t} \in W^{2, \infty}\left(\mathcal{F}_{0}\right)^{4}$ and $(\xi, \theta, t) \in$ $\mathcal{W} \times[0, \infty) \rightarrow \mathcal{J}_{\xi, \theta, t} \in W^{2, \infty}\left(\mathcal{F}_{0}\right)$ are of class $\mathcal{C}^{1}$, by Lemma 9 and by continuity of the product $W^{2, \infty}\left(\mathcal{F}_{0}\right) \times H^{2}\left(\mathcal{F}_{0}^{\prime}\right) \cap H^{1}\left(\mathcal{F}_{0}\right) \rightarrow H^{2}\left(\mathcal{F}_{0}^{\prime}\right) \cap H^{1}\left(\mathcal{F}_{0}\right)$, for $i=1,2$, the mapping

$$
\begin{gathered}
(\alpha, t, V) \in \mathcal{U} \times[0, \infty) \times \mathbb{H}^{2}\left(\mathcal{F}_{0}^{\prime}\right) \cap \mathbb{H}^{1}\left(\mathcal{F}_{0}\right) \\
\rightarrow \mathcal{J}_{\xi, \theta, t}^{-1} \nabla X_{\xi, \theta, t}^{(i)} \cdot\left[V+K_{\alpha, t}\right] \in H^{2}\left(\mathcal{F}_{0}^{\prime}\right) \cap H^{1}\left(\mathcal{F}_{0}\right)
\end{gathered}
$$

is of class $\mathcal{C}^{1}$. Recall that for $i=1,2, L_{i}(\xi, \theta, t)$ is defined by (39) (p. 13). Since the matrix inversion is a $\mathcal{C}^{\infty}$ operation from $G L_{2}(\mathbb{R})$ onto itself and since the derivation in Sobolev spaces is a bounded operator, we obtain that for $i=1,2$, the mapping

$$
(\alpha, t, V) \in \mathcal{U} \times[0, \infty) \times \mathbb{H}^{2}\left(\mathcal{F}_{0}^{\prime}\right) \cap \mathbb{H}^{1}\left(\mathcal{F}_{0}\right) \rightarrow L_{i}(\xi, \theta, t)\left[V+K_{\alpha, t}\right] \in \mathbb{H}^{1}\left(\mathcal{F}_{0}^{\prime}\right) \cap L^{2}\left(\mathcal{F}_{0}\right)^{2}
$$

is of class $\mathcal{C}^{1}$, and consequently, that the mapping

$$
\begin{aligned}
& (\alpha, t, V) \in \mathcal{U} \times[0, \infty) \times \mathbb{H}^{2}\left(\mathcal{F}_{0}^{\prime}\right) \cap \mathbb{H}^{1}\left(\mathcal{F}_{0}\right) \\
\rightarrow & \left(\operatorname{div}\left(L_{i}(\xi, \theta, t)\left[V+K_{\alpha, t}\right]\right)\right) \nabla X_{\xi, \theta, t}^{(i)} \in L^{2}\left(\mathcal{F}_{0}^{\prime}\right)^{2} \cap\left(\mathbb{H}_{\text {tan }}^{1}\right)^{\prime}\left(\mathcal{F}_{0}\right)
\end{aligned}
$$


is of class $\mathcal{C}^{1}$. By the same arguments, that the mapping

$$
\begin{aligned}
& (\alpha, t, P) \in \mathcal{U} \times[0, \infty) \times H^{1}\left(\mathcal{F}_{0}^{\prime}\right) \cap L^{2}\left(\mathcal{F}_{0}\right) \\
\rightarrow & \sum_{j} \partial_{j}\left(M_{i j}(\xi, \theta, t) P\right) \nabla X_{\xi, \theta, t}^{(i)} \in L^{2}\left(\mathcal{F}_{0}^{\prime}\right)^{2} \cap\left(\mathbb{H}_{\text {tan }}^{1}\right)^{\prime}\left(\mathcal{F}_{0}\right)
\end{aligned}
$$

is of class $\mathcal{C}^{1}$. Consequently, $Q$ is a $\mathcal{C}^{1}$ mapping from $\mathcal{U} \times[0, \infty) \times A$ into $L^{2}\left(\mathcal{F}_{0}^{\prime}\right)^{2} \cap$ $\left(\mathbb{H}_{\text {tan }}^{1}\right)^{\prime}\left(\mathcal{F}_{0}\right)$;

- $R$ is independant on the variable $(\alpha, t)$ and induces a bounded operator from $A$ into $H^{1}\left(\mathcal{F}_{0}^{\prime}\right) \cap L_{0}^{2}\left(\mathcal{F}_{0}\right)$. In particular, $R$ is of class $\mathcal{C}^{1}$.

- By definition, $\left(V^{*}, P^{*}\right)$ satisfies

$$
H\left(\alpha^{*}, t^{*} ; V^{*}, P^{*}\right)=0 .
$$

- Let us prove that the operator $\Lambda:=D_{(V, P)} H\left(\alpha^{*}, t^{*}, V^{*}, P^{*}\right)$ is a bicontinuous isomorphism from $A$ onto $B . \Lambda$ is defined for every $(V, P) \in A$ by

$$
\left\{\begin{aligned}
\Lambda(V, P) & =\left(\Lambda_{1}, \Lambda_{2}\right)(V, P) \\
\Lambda_{1}(V, P) & =\sum_{i}\left[-\nu \operatorname{div}\left(L_{i}^{*} V\right)+\sum_{j} \partial_{j}\left(M_{i j}^{*} P\right)\right] \nabla X^{(i)} \\
\Lambda_{2}(V, P) & =\operatorname{div} V
\end{aligned}\right.
$$

Clearly $\Lambda \in \mathcal{L}(A, B)$. We need to prove that $\Lambda$ is invertible with continuous inverse. Let $(f, g) \in B$. We need to prove that there exists one unique pair $(V, P) \in A$ such that

$$
(f, g)=\Lambda(V, P),
$$

that is, that there exists one unique solution to the problem

$$
\left\{\begin{aligned}
\sum_{i}\left[-\nu \operatorname{div}\left(L_{i}^{*} V\right)+\sum_{j} \partial_{j}\left(M_{i j}^{*} P\right)\right] \nabla X^{(i)}=f & \text { in } \mathcal{F}_{0}, \\
\operatorname{div} V=g & \text { in } \mathcal{F}_{0} .
\end{aligned}\right.
$$

Besides, we need to prove that this solution satisfies natural estimates with respect to $(f, g)$. To this end, we perform the opposite change of functions to (35) which transforms problem (49) posed on the reference domain $\mathcal{F}_{0}$ into a Stokes problem posed on $\mathcal{F}$. The existence and uniqueness of the solution to problem (49) result from the existence and uniqueness of the solution to the Stokes problem on $\mathcal{F}$, via transformation (35). Then we use classical estimates on the solution to the Stokes problem with slip boundary condition and the regularity of the diffeomorphism $X$ to control the norm of $(V, P)$ in $A$ by the norm of the source term $(f, g)$ in $B$.

We define the functions $v, p, \tilde{f}, \tilde{g}$ by the following relations:

$$
\left\{\begin{array}{l}
v \circ X=\mathcal{J}^{-1} J V \\
p \circ X=\mathcal{J}^{-1} P \\
\tilde{f} \circ X=\mathcal{J}^{-1} M^{*} f \\
\tilde{g} \circ X=\mathcal{J}^{-1} g
\end{array}\right.
$$


$(\tilde{f}, \tilde{g}) \in\left(\mathbb{H}_{\text {tan }}^{1}\right)^{\prime}(\mathcal{F}) \cap L^{2}\left(\mathcal{F}^{\prime}\right)^{2} \times H^{1}\left(\mathcal{F}^{\prime}\right) \cap L_{0}^{2}(\mathcal{F})$ and $(v, p)$ is solution to the following problem:

$$
\text { find }(v, p) \in \mathbb{H}_{\text {tan }}^{1}(\mathcal{F}) \times L_{0}^{2}(\mathcal{F})
$$

such that

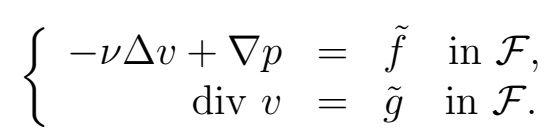

By the results in [32], problem (50) admits one unique solution, this solution has the regularity

$$
(v, p) \in \mathbb{H}^{2}\left(\mathcal{F}^{\prime}\right) \cap \mathbb{H}_{\text {tan }}^{1}(\mathcal{F}) \times H^{1}\left(\mathcal{F}^{\prime}\right) \cap L_{0}^{2}(\mathcal{F})
$$

and there exists a constant $C>0$ depending only on $\left(\alpha^{*}, t^{*}\right)$ such that

$$
\begin{gathered}
\|v\|_{\mathbb{H}^{2}\left(\mathcal{F}^{\prime}\right)}+\|v\|_{\mathbb{H}^{1}(\mathcal{F})}+\|p\|_{H^{1}\left(\mathcal{F}^{\prime}\right)}+\|p\|_{L^{2}(\mathcal{F})} \\
\leqslant C\left(\|\tilde{f}\|_{L^{2}\left(\mathcal{F}^{\prime}\right)^{2}}+\|\tilde{f}\|_{\left(\mathbb{H}_{\text {tan }}^{1}\right)^{\prime}(\mathcal{F})}+\|\tilde{g}\|_{H^{1}\left(\mathcal{F}^{\prime}\right)}+\|\tilde{g}\|_{L^{2}(\mathcal{F})}\right) .
\end{gathered}
$$

By construction of $X$, the partial derivatives of $X$ (resp. $X^{-1}$ ) up to order 2 are uniformly bounded on $\mathcal{F}_{0}$ (resp. $\mathcal{F}$ ). The domains $\mathcal{F}_{0}$ and $\mathcal{F}$ being bounded, there exist two constant $C_{1}, C_{2}>0$ (depending only on $\left(\alpha^{*}, t^{*}\right)$ ) such that

$$
\|(V, P)\|_{A} \leqslant C_{1}\left(\|v\|_{\mathbb{H}^{2}\left(\mathcal{F}^{\prime}\right)}+\|v\|_{\mathbb{H}^{1}(\mathcal{F})}+\|p\|_{H^{1}\left(\mathcal{F}^{\prime}\right)}+\|p\|_{L^{2}(\mathcal{F})}\right)
$$

and

$$
\|\tilde{f}\|_{L^{2}\left(\mathcal{F}^{\prime}\right)^{2}}+\|\tilde{f}\|_{\left(\mathbb{H}_{\text {tan }}^{1}\right)^{\prime}(\mathcal{F})}+\|\tilde{g}\|_{H^{1}\left(\mathcal{F}^{\prime}\right)}+\|\tilde{g}\|_{L^{2}(\mathcal{F})} \leqslant C_{2}\|(f, g)\|_{B}
$$

Finally, by inequalities (51)-(53), there exists a constant $C>0$ such that

$$
\|(V, P)\|_{A} \leqslant C\|(f, g)\|_{B}
$$

which proves that $\Lambda$ is invertible in $\mathcal{L}(A, B)$.

By the implicit function theorem, the mapping

$$
(\alpha, t) \in \mathcal{U} \times[0, \infty) \rightarrow\left(V_{\alpha, t}, P_{\alpha, t}\right) \in A
$$

solution to (48) is of class $\mathcal{C}^{1}$ in the neighborhood of $\left(\alpha^{*}, t^{*}\right)$. Since $\left(\alpha^{*}, t^{*}\right)$ is arbitrary, this mapping is of class $\mathcal{C}^{1}$ on $\mathcal{U} \times[0, \infty)$. Using Lemma 5 (p. 11), formula (35) (p. 12) and Lemma 9, we obtain

Proposition 11. The mapping

$$
(\alpha, t) \in \mathcal{U} \times[0, \infty) \rightarrow\left(u_{\alpha, t} \circ X_{\xi, \theta, t}, p_{\alpha, t} \circ X_{\xi, \theta, t}\right) \in \mathbb{H}^{2}\left(\mathcal{F}_{0}^{\prime}\right) \cap \mathbb{H}^{1}\left(\mathcal{F}_{0}\right) \times H^{1}\left(\mathcal{F}_{0}^{\prime}\right) \cap L_{0}^{2}\left(\mathcal{F}_{0}\right),
$$

where $\left(u_{\alpha, t}, p_{\alpha, t}\right)$ is the solution to problem (19), is of class $\mathcal{C}^{1}$. 
Proposition 4 results directly from this statement.

Step 3. Continuity of $F, G$ and local Lipschitz condition with respect to $\alpha$.

Recall that $F, G$ are defined by (24) (p. 8). Using the change of variable formula in the curvilinear integrals (see for instance [20] p. 190), we transform the integrals on $\partial \mathcal{S}(\xi, \theta, t)$ into integrals on the fixed boundary $\partial \mathcal{S}_{0}$. We denote by $\mathcal{J}_{\text {tan }}\left(X_{\xi, \theta, t}\right)$ the tangential jacobian of $X_{\xi, \theta, t}$ on $\partial \mathcal{S}_{0}$, defined by

$$
\mathcal{J}_{\text {tan }}\left(X_{\xi, \theta, t}\right)=\left|\left[J_{\xi, \theta, t}^{-1}\right]^{T} n_{0}\right| \mathcal{J}_{\xi, \theta, t}
$$

For $i=1,2$, we obtain

$$
\begin{aligned}
F^{(i)}(\alpha, t) & =-\frac{1}{m} \int_{\partial \mathcal{S}(\xi, \theta, t)}\left(\sigma\left(u_{\alpha, t}, p_{\alpha, t}\right)\right)^{(i)} \cdot n_{\xi, \theta, t} \mathrm{~d} s \\
& =-\frac{1}{m} \int_{\partial \mathcal{S}_{0}}\left(\sigma\left(u_{\alpha, t}, p_{\alpha, t}\right) \circ X_{\xi, \theta, t}\right)^{(i)} \cdot\left(n_{\xi, \theta, t} \circ X_{\xi, \theta, t}\right) \mathcal{J}_{t a n}\left(X_{\xi, \theta, t}\right) \mathrm{d} s .
\end{aligned}
$$

By formula (43) (p. 14), last equality can be written as follows:

$$
F^{(i)}(\alpha, t)=-\frac{1}{m} \int_{\partial \mathcal{S}_{0}} \mathcal{J}_{\xi, \theta, t}\left(J_{\xi, \theta, t}^{-1}\left(\sigma\left(u_{\alpha, t}, p_{\alpha, t}\right) \circ X_{\xi, \theta, t}\right)^{(i)}\right) \cdot n_{0} \mathrm{~d} s .
$$

As a consequence of Lemma 5 (p. 11) and Proposition 4, the mapping

$$
(\alpha, t) \in \mathcal{U} \times[0, \infty) \rightarrow \mathcal{J}_{\xi, \theta, t} J_{\xi, \theta, t}^{-1}\left(\sigma\left(u_{\alpha, t}, p_{\alpha, t}\right) \circ X_{\xi, \theta, t}\right)^{(i)} \in \mathbb{H}^{1}\left(\mathcal{F}_{0}^{\prime}\right)
$$

is of class $\mathcal{C}^{1}$. Since the trace operator from $\mathbb{H}^{1}\left(\mathcal{F}_{0}^{\prime}\right)$ into $L^{2}\left(\partial \mathcal{S}_{0}\right)^{2}$ and the inner product with the vector field $n_{0}$ in $L^{2}\left(\partial \mathcal{S}_{0}\right)^{2}$ are bounded and linear, we obtain that for $i=1$, 2 , the mapping

$$
(\alpha, t) \in \mathcal{U} \times[0, \infty) \rightarrow F^{(i)}(\alpha, t) \in \mathbb{R}
$$

is of class $\mathcal{C}^{1}$. In particular, $F$ is continuous and satisfies a local Lipshitz condition with respect to $\alpha$. The continuity of $G$ and the local Lipschitz condition result from the same argument, and from the facts that the mappings

$$
\xi \in \mathbb{R}^{2} \rightarrow(\cdot-\xi)^{\perp} \in L^{2}\left(\partial \mathcal{S}_{0}\right)
$$

and

$$
t \in[0, \infty) \rightarrow I(t) \in \mathbb{R}
$$

are of class $\mathcal{C}^{1}$.

Step 4. Rewriting of problem (25) as a first order differential system.

Problem (25) (p.9) is equivalent to the following: find $T>0$ and a function $\alpha \in$ $\mathcal{C}^{2}\left((0, T), \mathbb{R}^{6}\right) \cap \mathcal{C}^{1}\left([0, T), \mathbb{R}^{6}\right)$ such that

$$
\left\{\begin{aligned}
\alpha^{\prime}(t) & =\mathcal{A} \alpha(t)+\mathcal{H}(\alpha(t), t) \quad \forall t \in(0, T), \\
\alpha(0) & =\alpha_{0}
\end{aligned}\right.
$$


where $\alpha_{0}=\left(0,0, \dot{\xi}_{0}, \dot{\theta}_{0}\right), \mathcal{A} \in \mathcal{M}_{6}(\mathbb{R})$ and $\mathcal{H}$ are defined by

$$
\mathcal{A}=\left(\begin{array}{cccccc}
0 & 0 & 0 & 1 & 0 & 0 \\
0 & 0 & 0 & 0 & 1 & 0 \\
0 & 0 & 0 & 0 & 0 & 1 \\
0 & 0 & 0 & 0 & 0 & 0 \\
0 & 0 & 0 & 0 & 0 & 0 \\
0 & 0 & 0 & 0 & 0 & 0
\end{array}\right), \quad \mathcal{H}=\left(\begin{array}{c}
0 \\
0 \\
0 \\
F^{(1)} \\
F^{(2)} \\
G
\end{array}\right)
$$

By the results in Section 4, system (55) satisfies the assumptions of the Cauchy-Lipschitz theorem. This proves the first two points in Theorem 1. The third point is a consequence of the result by Hillairet [21].

\section{$5 \quad$ Proof of Theorem 2.}

To study the stability of the trajectories with respect to the deformation $\Theta^{*}$, we treat $\Theta^{*}$ as a parameter in the Cauchy problem (55). We use the same notations as in Section 4. We consider the open subset $\mathcal{U} \subset \mathbb{R}^{6}$ defined by (18) (p. 7) and define the following Cauchy problem with parameter $\Theta^{*}$ :

$$
\left\{\begin{aligned}
\alpha^{\prime}(t) & =L\left(\alpha(t), t, \Theta^{*}\right), \\
\alpha(0) & =\alpha_{0},
\end{aligned}\right.
$$

where $\alpha_{0} \in \mathcal{U}$ is a fixed initial datum and $L$ is defined by

$$
L\left(\alpha, t, \Theta^{*}\right)=\mathcal{A} \alpha+\mathcal{H}\left(\alpha, t, \Theta^{*}\right), \quad\left(\alpha, t, \Theta^{*}\right) \in \mathcal{U} \times[0, \infty) \times \mathcal{D} .
$$

Here we have treated $\Theta^{*}$ as a variable in the definition of $\mathcal{H}$ given in Section 4. (Recall that $X_{\xi, \theta, t}$ depends on $\Theta^{*}$ through formulas (28) and (32).) Adapting the method in Section 4 one can easily prove that $L$ is continuous on $\mathcal{U} \times[0, \infty) \times \mathcal{D}$.

Now we can prove Theorem 2. Let $\Theta^{*} \in \mathcal{D}$ be a given deformation and $\left(\Theta_{n}^{*}\right)_{n \in \mathbb{N}}$ be a sequence of deformations converging to $\Theta^{*}$ in $\mathcal{D}$. We denote by $(C P)_{\Theta^{*}}$ the Cauchy problem associated to $\Theta^{*}$ with initial datum $\alpha_{0}$, and by $\alpha$ the maximal solution to $(C P)_{\Theta^{*}}$. For every $n \in \mathbb{N}$, we denote by $(C P)_{\Theta_{n}^{*}}$ the Cauchy problem associated to $\Theta_{n}^{*}$ with the same initial datum $\alpha_{0}$, and by $\alpha^{n}$ the maximal solution to $(C P)_{\Theta_{n}^{*}}$. By a standard topology argument, the set

$$
\mathcal{K}=\left\{\Theta_{n}^{*}, n \in \mathbb{N}^{*}\right\} \cup\left\{\Theta^{*}\right\}
$$

is a compact subset of $\mathcal{D}$. Since $\mathcal{U}$ is open, there exists $r_{0}>0$ such that

$$
\bar{B}\left(\alpha_{0}, r_{0}\right) \subset \mathcal{U}
$$

Let us fix $\tau>0$ and define $V_{0} \subset \mathcal{U} \times[0, \infty) \times \mathcal{K}$ by

$$
V_{0}=\bar{B}\left(\alpha_{0}, r_{0}\right) \times[0, \tau] \times \mathcal{K} .
$$


By continuity of $L$, there exists $M>0$ such that

$$
\|L(\alpha, t, \chi)\| \leqslant M, \quad(\alpha, t, \chi) \in V_{0} .
$$

Now fix $T_{1}>0$ such that

$$
T_{1}<\min \left(\tau, \frac{r_{0}}{M}\right)
$$

By (56)-(57) and by the Cauchy-Peano theorem, for every $n \in \mathbb{N},(C P)_{\Theta^{*}}$ and $(C P)_{\Theta_{n}^{*}}$ admit a solution defined on $\left[0, T_{1}\right]$. By the results in Section 4 , these solutions are unique on $\left[0, T_{1}\right]$, and by maximality of $\alpha, \alpha^{n}$, these solutions necessarily coincide with $\alpha, \alpha^{n}$ on $\left[0, T_{1}\right]$, which yields

$$
\alpha(t), \alpha_{n}(t) \in \bar{B}\left(\alpha_{0}, r_{0}\right) \quad \forall n \in \mathbb{N}, \quad \forall t \in\left[0, T_{1}\right] .
$$

This proves the first point in Theorem 2. Now let $T^{*}>0$ such that the solutions $\left(\xi_{n}, \theta_{n}\right)$ are well-defined on $\left[0, T^{*}\right]$ for all $n \in \mathbb{N}$. Since $L$ is continuous on the compact $\mathcal{K}$, there exists a continuous function

$$
\eta: \mathbb{R}^{+} \rightarrow \mathbb{R}^{+}
$$

such that

$$
\left\|L\left(\alpha^{n}(t), t, \Theta_{n}^{*}\right)-L\left(\alpha^{n}(t), t, \Theta^{*}\right)\right\| \leqslant \eta\left(\left\|\Theta_{n}^{*}-\Theta^{*}\right\|_{\mathcal{D}}\right), \quad t \in\left[0, T^{*}\right]
$$

and $\lim _{u \rightarrow 0^{+}} \eta(u)=0$. As a result, for every $n \in \mathbb{N}, \alpha^{n}$ is an $\varepsilon_{n^{-}}$approximate solution to $\left(C P_{\Theta^{*}}\right)$, with

$$
\varepsilon_{n}=\eta\left(\left\|\Theta_{n}^{*}-\Theta^{*}\right\|_{\mathcal{D}}\right) .
$$

By the results in Section 4, we can suppose that there exists $k>0$ (depending on $\Theta^{*}$ ) such that the mapping

$$
(\alpha, t) \in \bar{B}\left(\alpha_{0}, r_{0}\right) \times\left[0, T^{*}\right] \rightarrow L\left(\alpha, t, \Theta^{*}\right) \in \bar{B}\left(\alpha_{0}, r_{0}\right)
$$

is $k$-lipschitzian with respect to $\alpha$. As a result, by Gronwall lemma (see for instance [14]),

$$
\left\|\alpha^{n}(t)-\alpha(t)\right\| \leqslant \varepsilon_{n} \frac{e^{k t}-1}{k}, \quad t \in\left[0, T^{*}\right],
$$

which yields

$$
\sup _{t \in\left[0, T^{*}\right]}\left\|\alpha^{n}(t)-\alpha(t)\right\| \leqslant \frac{e^{k T^{*}}-1}{k} \eta\left(\left\|\Theta_{n}^{*}-\Theta^{*}\right\|_{\mathcal{D}}\right) .
$$

Taking the limit when $n \rightarrow \infty$, we get the second point in Theorem 2 .

Acknowledgements. The author gratefully acknowledge the anonymous referee for his careful reading and for a number of useful suggestions that have been incorporated in the current version of the manuscript. 


\section{References}

[1] François Alouges, Antonio DeSimone, and Aline Lefebvre. Optimal strokes for low Reynolds number swimmers: an example. J. Nonlinear Sci., 18(3):277-302, 2008.

[2] H. Beirão Da Veiga. Regularity for Stokes and generalized Stokes systems under nonhomogeneous slip-type boundary conditions. Adv. Differential Equations, 9(9-10):10791114, 2004.

[3] Juan Antonio Bello, Enrique Fernández-Cara, Jérôme Lemoine, and Jacques Simon. The differentiability of the drag with respect to the variations of a Lipschitz domain in a Navier-Stokes flow. SIAM J. Control Optim., 35(2):626-640, 1997.

[4] H.C. Berg and R. Anderson. Bacteria swim by rotating their flagellar filament. Nature, 245:380-382, 1973.

[5] M. E. Bogovskil. Solution of the first boundary value problem for an equation of continuity of an incompressible medium. Dokl. Akad. Nauk SSSR, 248(5):1037-1040, 1979.

[6] Muriel Boulakia. Existence of weak solutions for the motion of an elastic structure in an incompressible viscous fluid. C. R. Math. Acad. Sci. Paris, 336(12):985-990, 2003.

[7] Alberto Bressan. Impulsive control of Lagrangian systems and locomotion in fluids. Discrete Contin. Dyn. Syst., 20(1):1-35, 2008.

[8] Dorin Bucur, Eduard Feireisl, Šárka Nečasová, and Joerg Wolf. On the asymptotic limit of the Navier-Stokes system on domains with rough boundaries. J. Differential Equations, 244(11):2890-2908, 2008.

[9] Juan Casado-Díaz, Enrique Fernández-Cara, and Jacques Simon. Why viscous fluids adhere to rugose walls: a mathematical explanation. J. Differential Equations, 189(2):526-537, 2003.

[10] Antonin Chambolle, Benoît Desjardins, Maria J. Esteban, and Céline Grandmont. Existence of weak solutions for the unsteady interaction of a viscous fluid with an elastic plate. J. Math. Fluid Mech., 7(3):368-404, 2005.

[11] Alexandre Cherman, Joaquin Delgado, Fernando Duda, Kurt Ehlers, Jair Koiller, and Richard Montgomery. Low Reynolds number swimming in two dimensions. In Hamiltonian systems and celestial mechanics (Pátzcuaro, 1998), volume 6 of World Sci. Monogr. Ser. Math., pages 32-62. World Sci. Publ., River Edge, NJ, 2000.

[12] Daniel Coutand and Steve Shkoller. Motion of an elastic solid inside an incompressible viscous fluid. Arch. Ration. Mech. Anal., 176(1):25-102, 2005.

[13] Daniel Coutand and Steve Shkoller. The interaction between quasilinear elastodynamics and the Navier-Stokes equations. Arch. Ration. Mech. Anal., 179(3):303-352, 2006. 
[14] J.-P. Demailly. Analyse numérique et équations différentielles. Presses Universitaires de Grenoble, 2006.

[15] B. Desjardins and M. J. Esteban. Existence of weak solutions for the motion of rigid bodies in a viscous fluid. Arch. Ration. Mech. Anal., 146(1):59-71, 1999.

[16] B. Desjardins, M. J. Esteban, C. Grandmont, and P. Le Tallec. Weak solutions for a fluid-elastic structure interaction model. Rev. Mat. Complut., 14(2):523-538, 2001.

[17] Eduard Feireisl. On the motion of rigid bodies in a viscous incompressible fluid. $J$. Evol. Equ., 3(3):419-441, 2003.

[18] Giovanni P. Galdi. An introduction to the mathematical theory of the Navier-Stokes equations. Vol. I, volume 38 of Springer Tracts in Natural Philosophy. Springer-Verlag, New York, 1994. Linearized steady problems.

[19] Max D. Gunzburger, Hyung-Chun Lee, and Gregory A. Seregin. Global existence of weak solutions for viscous incompressible flows around a moving rigid body in three dimensions. J. Math. Fluid Mech., 2(3):219-266, 2000.

[20] Antoine Henrot and Michel Pierre. Variation et optimisation de formes, volume 48 of Mathématiques et Applications (Berlin) [Mathematics and Applications]. Springer, Berlin, 2005. Une analyse géométrique. [A geometric analysis].

[21] M. Hillairet. Lack of collision between solid bodies in a 2D incompressible viscous flow. Comm. Partial Differential Equations, 32(7-9):1345-1371, 2007.

[22] Atsushi Inoue and Minoru Wakimoto. On existence of solutions of the Navier-Stokes equation in a time dependent domain. J. Fac. Sci. Univ. Tokyo Sect. IA Math., 24(2):303-319, 1977.

[23] Dorina Mitrea, Marius Mitrea, and Sylvie Monniaux. The Poisson problem for the exterior derivative operator with Dirichlet boundary condition in nonsmooth domains. Commun. Pure Appl. Anal., 7(6):1295-1333, 2008.

[24] Alexandre Munnier. On the self-displacement of deformable bodies in a potential fluid flow. Math. Models Methods Appl. Sci., 18(11):1945-1981, 2008.

[25] François Murat and Jacques Simon. Étude de problèmes d'optimal design, volume 41 of Lecture Notes in Computer Science, pages 54-62. Springer Berlin / Heiderberg, 1976.

[26] E.M. Purcell. Life at low reynolds number. Am. J. Phys., 45:3-11, 1977.

[27] Jorge San Martín, Jean-François Scheid, Takéo Takahashi, and Marius Tucsnak. An initial and boundary value problem modeling of fish-like swimming. Arch. Ration. Mech. Anal., 188(3):429-455, 2008. 
[28] Jorge Alonso San Martín, Victor Starovoitov, and Marius Tucsnak. Global weak solutions for the two-dimensional motion of several rigid bodies in an incompressible viscous fluid. Arch. Ration. Mech. Anal., 161(2):113-147, 2002.

[29] Alfred Shapere and Frank Wilczek. Geometry of self-propulsion at low Reynolds number. J. Fluid Mech., 198:557-585, 1989.

[30] Jacques Simon. Domain variation for drag in Stokes flow. In Control theory of distributed parameter systems and applications (Shanghai, 1990), volume 159 of Lecture Notes in Control and Inform. Sci., pages 28-42. Springer, Berlin, 1991.

[31] Jan Sokołowski and Jean-Paul Zolésio. Introduction to shape optimization, volume 16 of Springer Series in Computational Mathematics. Springer-Verlag, Berlin, 1992. Shape sensitivity analysis.

[32] V. A. Solonnikov and V. E. Ščadilov. On a boundary value problem for a stationary system of navier-stokes equations. Proc. Steklov Inst. Math., 125:186-199, 1973.

[33] Geoffrey Taylor. Analysis of the swimming of microscopic organisms. Proc. Roy. Soc. London. Ser. A., 209:447-461, 1951.

[34] M. Tryantafyllou and G. Tryantafyllou. An efficient swimming machine. Scientific American, 272:64-70, 1995. 